\title{
Dynamic Regulation of Caveolin-1 Phosphorylation and Caveolae Formation by Mammalian Target of Rapamycin Complex 2 in Bladder Cancer Cells
}

Andrew M. Hau, * Sounak Gupta, ${ }^{*}$ Mariah Z. Leivo, ${ }^{*}$ Kazufumi Nakashima, ${ }^{*}$ Jesus Macias, ${ }^{*}$ Weidong Zhou, ${ }^{\dagger}$ Alex Hodge, Julie Wulfkuhle, ${ }^{\dagger}$ Brian Conkright, ${ }^{\ddagger}$ Krithika Bhuvaneshwar, ${ }^{\ddagger}$ Shruti Rao, ${ }^{\ddagger}$ Subha Madhavan, ${ }^{\ddagger}$ Emanuel F. Petricoin III, ${ }^{\dagger}$ and Donna E. Hansel*

From the Department of Pathology,* University of California, San Diego, La Jolla, California; the Center for Applied Proteomics and Personalized Medicine, ${ }^{\dagger}$ George Mason University, Manassas, Virginia; and The Innovation Center for Biomedical Informatics, ${ }^{\ddagger}$ Georgetown University, Washington, District of Columbia

Accepted for publication

May 7, 2019.

Address correspondence to Donna E. Hansel, M.D., Ph.D., Department of Pathology, University of California, San Diego, 9500 Gilman Dr., MC 0612, La Jolla, CA 92093. E-mail: dhansel@ ucsd.edu.

\begin{abstract}
The mammalian target of rapamycin (mTOR) and associated phosphatidylinositol 3-kinase/AKT/mTOR signaling pathway is commonly up-regulated in cancer, including bladder cancer. mTOR complex 2 (mTORC2) is a major regulator of bladder cancer cell migration and invasion, but the mechanisms by which mTORC2 regulates these processes are unclear. A discovery mass spectrometry and reverse-phase protein array-based proteomics dual approach was used to identify novel mTORC2 phosphoprotein targets in actively invading cancer cells. mTORC2 targets included focal adhesion kinase, protooncogene tyrosine-protein kinase Src, and caveolin-1 (Cav-1), among others. Functional testing shows that mTORC2 regulates Cav-1 localization and dynamic phosphorylation of Cav-1 on Y14. Regulation of Cav-1 activity by mTORC2 also alters the abundance of caveolae, which are specialized lipid raft invaginations of the plasma membrane associated with cell signaling and membrane compartmentalization. Our results demonstrate a unique role for mTORC2-mediated regulation of caveolae formation in actively migrating cancer cells. (Am J Pathol 2019, 189: 1846-1862; https://doi.org/10.1016/ j.ajpath.2019.05.010)
\end{abstract}

Bladder cancer is the most common urinary tract malignancy, with an estimated 81,000 new bladder cancer cases and 17,000 bladder cancer-associated deaths this year alone in the United States. ${ }^{1}$ Although most patients present with noninvasive disease, progression to invasive cancer occurs in approximately $40 \%$ to $60 \%$ of patients and results in an increased risk of metastasis and reduced diseasespecific survival. ${ }^{2}$ Current treatment modalities include bladder removal with neoadjuvant chemotherapy or radiation therapy for locally advanced, invasive disease and chemotherapy or immunotherapy for metastatic disease. ${ }^{3}$ Understanding the cell signaling pathways relevant to the development of invasive bladder cancer behavior may enhance the ability to develop strategic approaches in bladder cancer therapy.
The mammalian target of rapamycin complex 2 (mTORC2) is a major protein complex that drives bladder cancer invasion and key effector of the mTOR pathway. ${ }^{4-6}$ mTOR is an evolutionary conserved serine/threonine kinase that can integrate extracellular and environmental cues, such as growth factor signaling and nutrient status, to affect a diverse array of cell processes, such as cell proliferation,

\footnotetext{
Supported by the Department of Pathology, University of California, San Diego.

Disclosures: E.F.P. has US government- and George Mason University-assigned patents concerning the reverse-phase protein array technology and receives licensing and royalty distribution from these patents. E.F.P. is a consultant to ADVX Investors Group, LLC, which has licenses in reverse-phase protein array-based immunoprecipitation from George Mason University and US government-owned patents.
} 
metabolism, and motility. Specifically, mTOR mediates these functions as an essential component of two multiprotein complexes, mTORC1 and mTORC2. These complexes can be distinguished by their subunit composition (raptor and PRAS40 for mTORC1 and rictor, stress-activated map kinase interacting protein 1 (mSin 1$)$, and protor for mTORC2) and their downstream functions. ${ }^{7}$ Whereas mTORC1 regulates cell growth and metabolism through phosphorylation of p70-S6K and 4EBP1, mTORC2 phosphorylates AKT S473, increases Rho-GTPase activity, and activates protein kinase $\mathrm{C}$ (PKC) ${ }^{7,8}$ We previously described a promigratory and proinvasive function of mTORC2 on in vitro bladder cancer cell migration and invasion, which was mediated through Rho-GTPase activation, ${ }^{4}$ as well as increased mTORC2 activity occurring in invasive, high-stage human bladder cancers. Given the important role of $\mathrm{mTORC} 2$ in regulating bladder cancer cell migration and invasion, a proteomics-based approach using mass spectrometry (MS) and reverse-phase protein array (RPPA) was used to discover and identify novel targets of mTORC2 signaling in motile and nonmotile conditions.

Phosphopeptide enrichment coupled to MS and RPPA represent two powerful complementary phosphoproteomic approaches that allow for discovery-based, quantifiable detection of proteins within biological samples. Although these technologies are able to effectively uncover important signaling events and map the activated signaling architecture of input samples, each have their own limitations. ${ }^{9}$ Although an MS approach can provide a global unbiased view of phosphoprotein expression, it is also possible that important signaling-related phosphoproteins may be undetected because of low relative protein abundance within a sample, low stoichiometry of phosphorylated signaling proteins compared with phosphorylated high abundance proteins and/or rapid degradation or dephosphorylation of a protein before analysis. By contrast, RPPA has greater analytical sensitivity than MS because of its ability to measure the phosphorylation state of very low abundance signaling proteins from microscopic quantities of cells. However, the biggest limitation of RPPA is the availability and dependence of antibodies for detection of proteins. Furthermore, RPPA can be viewed as a somewhat biased approach because arrays of specific antibodies are selected to probe a biological sample(s) for changes in protein expression and/or activity. Nevertheless, both MS and RPPA serve as increasingly important and complementary technologies for protein signaling analysis in cancer and other diseases.

Here, a parallel MS- and RPPA-based proteomics discovery approach was used to elucidate novel downstream targets of mTORC2 signaling in motile bladder cancer cells, followed by validation and functional testing of a subset of protein signaling networks that appeared relevant for migration and invasion. Multiple classes of proteins regulated by mTORC2, including mediators of cell morphology, cell assembly and organization, cell adhesion, cytoskeletal rearrangement, and cell motility, were identified. A subset of putative mTORC2-mediated targets were validated, and the ability of $\mathrm{mTORC} 2$ to regulate dynamic phosphorylation of caveolin-1 (Cav-1) on Y14 and Cav-1 cellular localization, both of which represent novel downstream effects of mTORC2, was functionally tested. Furthermore, mTORC2 can also induce up-regulation of cavin1 , an essential component for caveolae formation and function. The results of this study have identified a novel role for mTORC2-regulation of caveolae formation during bladder cancer cell motility.

\section{Materials and Methods}

\section{Cell Culture}

Human J82 and T24 bladder cancer cells were obtained and authenticated from the ATCC (Manassas, VA). Cells were grown in RPMI 1640 medium (Thermo Fisher Scientific, Grand Island, NY) supplemented with $10 \%$ fetal bovine serum (Gibco) and maintained at $37^{\circ} \mathrm{C}$ in a humidified chamber containing $5 \% \mathrm{CO}_{2}$.

\section{Gene Silencing and Serum Stimulation}

SMARTpool siGENOME siRNA against RICTOR (siRictor; catalog number M-016984-02) and CAVI (catalog number M-003467-01) were purchased from GE Dharmacon (Lafayette, $\mathrm{CO}$ ) and transfected into cells using Lipofectamine RNAiMAX reagent (Invitrogen, Carlsbad, CA). Pooled siGENOME nontargeting control siRNA (siNTC) numbers 2-5 (catalog numbers D-001210-02 to -05) was used for controls. Briefly, cells were plated onto 100-mm dishes and incubated overnight. Each plate of cells was transfected in Opti-MEM reduced serum media (Gibco) containing siRNA (160 pmol) and maintained for 72 hours. For serum stimulation, media was aspirated from plates and cells were washed three times with phosphate-buffered saline (PBS) before the addition of RPMI 1640 medium supplemented with $10 \%$ fetal bovine serum during the last hour of gene silencing.

\section{Immunoblotting}

Whole cell extracts were prepared using radioimmunoprecipitation assay buffer $(25 \mathrm{mmol} / \mathrm{L}$ Tris hydrochloride, $\mathrm{pH} 7.6,150 \mathrm{mmol} / \mathrm{L} \mathrm{NaCl}, 1 \%$ Triton X-100, $0.5 \%$ sodium deoxycholate, $0.1 \%$ SDS) containing PhosSTOP phosphatase and cOmplete Mini EDTA-free protease inhibitor cocktails (Roche, Mannheim, Germany). Cell lysates were cleared by centrifugation at $16,000 \times g$ for 15 minutes at $4^{\circ} \mathrm{C}$, and protein concentration was determined by the bicinchoninic acid method. Equal amounts $(10 \mu \mathrm{g})$ of protein were separated by SDS-PAGE using $4 \%$ to $15 \%$ Tris-Glycine Gradient gels (Bio-Rad, Hercules, PA) and immobilized onto polyvinylidene fluoride membranes. Membranes were blocked in 5\% (w/v) nonfat dry milk in 50 
$\mathrm{mmol} / \mathrm{L}$ Tris hydrochloride, $\mathrm{pH} 7.4,150 \mathrm{mmol} / \mathrm{L} \mathrm{NaCl}$ (TBS) plus $0.1 \%$ Tween-20 (TBS-T) and then incubated at $4{ }^{\circ} \mathrm{C}$ overnight with the appropriate primary antibody diluted in 5\% (w/v) nonfat dry milk or bovine serum albumin. The next day, membranes were washed with TBS-T (three times for 5 minutes each) then incubated with the appropriate horseradish peroxidase-conjugated secondary antibody for 1 hour at room temperature. Membranes were washed again in TBS-T (three times for 5 minutes each) and proteins detected using Clarity Western ECL blotting substrate (BioRad). Primary antibodies for 4EBP1 (1:1000; catalog number 9644), pan-AKT (1:1000; catalog number 4685), autocrine motility factor receptor $(1: 1000$; catalog number 9590), Cav-1 (1:1000; catalog number 3267), cofilin (1:1000; catalog number 5175), epidermal growth factor receptor (EGFR) (1:1000; catalog number 4267), focal adhesion kinase (FAK; 1:1000; catalog number 13009), human epidermal growth factor receptor 2 HER2; 1:1000; catalog number 4290), mTOR (1:1000; catalog number 2983), N-cadherin (1:1000; catalog number 13116), phosphorylated (p-) 4EBP1 T37/46 (1:2000; catalog number 2855), p-AKT S473 (1:2000; catalog number 4060), p-AKT T308 (1:1000; catalog number 4056), p-CAV-1 Y14 (1:1000; catalog number 3251), p-cofilin S3 (1:1000; catalog number 3313), p-EGFR Y1173 (1:1000; catalog number 4407), p-ezrin T567/radixin T564/moesin T558 (1:1000; catalog number 3141), p-FAK Y397 (1:1000; catalog number 8556), p-FAK Y567/577 (1:1000; catalog number 3281), p-FAK Y925 (1:1000; catalog number 3284), pHER2 Y1248 (1:1000; catalog number 2247), p-HER2 Y877 (1:1000; catalog number 2241), p-mTOR S2448 (1:1000; catalog number 5536), p-p70-S6K T389 (1:1000; catalog number 9234), $\mathrm{p}$-platelet-derived growth factor receptor (p-PDGFR)- $\alpha$ Y754 (1:1000; catalog number 2992), p-PDGFR- $\beta$ Y751 (1:1000; catalog number 4549), p-S6 S235/236 (1:2000; catalog number 4858), p-S6 S240/ 244 (1:2000; catalog number 5364), p-SRC Y527 (1:1000; catalog number 2105), p-SRC Y416 (1:1000; catalog number 6943), p-Talin S425 (1:1000; catalog number 5426), p-vascular endothelial growth factor receptor 2 Y1175 (1:1000; catalog number 3770), p70-S6K (1:1000; catalog number 2708), rictor (1:1000; catalog number 2140), S6 (1:2000; catalog number 2217), snail (1:1000; catalog number 3879), SRC (1:1000; catalog number 2123), zonula occludens-1 (ZO-1; 1:1000; catalog number 8193), and $\beta$-catenin $(1: 1000$; catalog number 8480$)$ were purchased from Cell Signaling (Danvers, MA). Primary antibodies for liprin $\alpha-1$ (1:1000; catalog number ab26192), liprin $\beta-1$ (1:1000; catalog number ab104117), and cavin-1 (1:1000; catalog number ab48824) were purchased from AbCam (Cambridge, UK). Primary antibodies for p-Adducin S662 (1:1000; catalog number 06-820) and mSin1 (1:1000; catalog number NBP1-89569) were purchased from EMD Millipore (Burlington, MA) and Novus (Centennial, CO), respectively. Actin antibody (1:5000; Sigma-Aldrich, St. Louis, MO) was routinely used as a loading control. Densitometry analysis was performed using ImageJ software version 1.48 (NIH, Bethesda, MD; http:// imagej.nih.gov/ij).

\section{Immunoprecipitation of Rictor}

Immunoprecipitation of rictor-containing mTOR complexes was performed as previously described with minor modification. ${ }^{10}$ T24 and J82 cells were lyzed for 30 minutes on ice with $0.3 \%$ CHAPS lysis buffer (40 mmol/L HEPES, $\mathrm{pH}$ $7.5,120 \mathrm{mmol} / \mathrm{L} \mathrm{NaCl}$, and $1 \mathrm{mmol} / \mathrm{L}$ EDTA) containing protease and phosphatase inhibitors. After centrifugation at $16,000 \times g$ for 15 minutes, fresh whole cell lysates $(1 \mathrm{mg})$ were incubated with $3 \mu \mathrm{g}$ of rictor (catalog number 5379; Cell Signaling) or $3 \mu \mathrm{g}$ of rabbit IgG isotype control Sepharose bead conjugates (catalog number 3423; Cell Signaling) for 2 hours with rotation at $4^{\circ} \mathrm{C}$. Beads were then collected by centrifugation at $2000 \times g$ for 5 minutes and washed three times with lysis buffer. The captured immunoprecipitates were eluted by heating in $35 \mu \mathrm{L} 2 \times$ Laemmli sample buffer containing $50 \mathrm{mmol} / \mathrm{L}$ 2-mercaptoethanol for 5 minutes at $95^{\circ} \mathrm{C}$ and then analyzed by immunoblot analysis. Co-immunoprecipitation of endogenous Cav-1 was detected with a monoclonal mouse anti-Cav-1 antibody (catalog number MAB5736; R\&D Systems, Minneapolis, MN).

\section{Cell Migration Assay}

Modified scratch-wound migration assays were performed as previously described. ${ }^{4}$

\section{Phosphoprotein Enrichment and MS}

Phosphoproteomic analysis of cell lysates was performed as previously described. ${ }^{11}$ Cell lysates were prepared by resuspension for 1 hour in lysis buffer consisting of Tris hydrochloride $(50 \mathrm{mmol} / \mathrm{L}, \mathrm{pH} 7.4), \mathrm{NaCl}(150 \mathrm{mmol} / \mathrm{L})$, Triton X-100 (0.5\% w/v), NP-40 (0.5\% w/v), $80 \mathrm{mmol} / \mathrm{L}$ dithiothreitol, $10 \mu \mathrm{L} / \mathrm{mL}$ of protease inhibitor cocktails (Sigma-Aldrich), $1 \mathrm{mmol} / \mathrm{L}$ phenylmethylsulfonyl fluoride, $1 \mathrm{mmol} / \mathrm{L} \mathrm{Na} \mathrm{VO}_{4}$, and PhosSTOP phosphatase inhibitor cocktail (Roche, Mannheim, Germany), sonicated for 30 seconds, and centrifuged at $16,000 \times g$ for 10 minutes. The supernatants were precipitated with 4 volumes of acetone (Sigma-Aldrich) overnight at $-20^{\circ} \mathrm{C}$ and centrifuged at $9000 \times g$ for 5 minutes. The pellets were dried by lyophylization (Heto, Dry Winner) for 2 hours.

The cell pellets were resuspended in $200 \mu \mathrm{L}$ of $8 \mathrm{~mol} / \mathrm{L}$ urea, and protein concentrations were measured by Bradford Assay (Bio-Rad). Protein samples were then reduced with $10 \mathrm{mmol} / \mathrm{L}$ dithiothreitol for 30 minutes at $37^{\circ} \mathrm{C}$ and then alkylated by $50 \mathrm{mmol} / \mathrm{L}$ iodoacetamide for 20 minutes at room temperature. The concentrated urea in the sample was diluted to a final concentration of $2 \mathrm{~mol} / \mathrm{L}$, and the proteins were digested by trypsin at $37^{\circ} \mathrm{C}$ for 6 hours in a buffer 
containing ammonium bicarbonate $(50 \mathrm{mmol} / \mathrm{L}, \mathrm{pH}$ 9). The digestion mixture was then acidified by adding glacial acetic acid to a final concentration of $2 \%$ and desalted by SepPak $\mathrm{C}_{18}$ column (Waters Corp., Milford, MA).

Phosphopeptides were enriched from the desalted 1-mg tryptic peptides using $\mathrm{TiO}_{2}$ column $(200 \mu \mathrm{m} \times 2 \mathrm{~cm})$ packed in-house. ${ }^{11}$ A total of $100 \mathrm{fmol}$ of standard phosphopeptide angiotensin II phosphate was added to the SepPak-cleaned sample. The sample was then mixed with an equal volume of loading buffer $(200 \mathrm{mg} / \mathrm{mL}$ DHB, $5 \%$ trifluoroacetic acid, $80 \%$ acetonitrile), and loaded into the $\mathrm{TiO}_{2}$ column using the Pressure Cell (Brechbühler Inc., Schlieren, Switzerland) with flow rate of $3 \mu \mathrm{L} /$ minute. The column was washed by $200 \mu \mathrm{L}$ of Wash Buffer $1(40 \mathrm{mg} /$ $\mathrm{mL}$ dihydroxybenzoic acid, $2 \%$ trifluoroacetic acid, $80 \%$ acetonitrile) and $2 \times 200 \mu \mathrm{L}$ of a second wash buffer $2(2 \%$ trifluoroacetic acid, 50\% acetonitrile) to remove nonphosphopeptides. Phosphopeptides were eluted from the column with the elution buffer (5\% ammonia solution). Ammonia in the eluate was removed by lyophilization (approximately 3 minutes), and the sample was acidified by adding glacial acetic acid to a final concentration of $2 \%$ and desalted by ZipTip (EMD Millipore).

The purified phosphopeptides were analyzed by highsensitive reversed-phase liquid chromatography coupled nanospray tandem MS using an LTQ-Orbitrap mass spectrometer (Thermo Fisher Scientific). LTQ-Orbitrap provides high-accuracy mass measurement that is essential for the validation of modified peptide identification and the reduction of false-positive identification. The reversed-phase LC column was slurry-packed in house with $5 \mu \mathrm{m}$ of $200 \AA$ pore size $\mathrm{C}_{18}$ resin (Michrom BioResources, Inc., Auburn, $\mathrm{CA})$ in a $100 \mu \mathrm{m}$ i.d. $\times 10$-cm-long piece of fused silica capillary (Polymicro Technologies, Phoenix, AZ) with a laser-pulled tip. After packing, the new column, the highperformance liquid chromatography system (Surveyor MS Pump Plus from Thermo Fisher Scientific), and the LTQOrbitrap were tested by analyzing 100 fmol of Yeast Enolase Standard \& Tryptic Digestion (catalog number PTD/00001/46; Michrom Bioresources, Inc.) to ensure that stable electrospray ionization, desired mass accuracy, peak resolution, peak intensity, and retention time could be obtained. Additional iteration was performed to ensure reproducibility. A total of $100 \mathrm{fmol}$ of standard peptide angiotensin I was spiked into the sample as an internal standard. After sample injection, the column was washed for 5 minutes with mobile phase A ( $0.1 \%$ formic acid), and peptides were eluted using a linear gradient of $0 \%$ mobile phase B (0.1\% formic acid, $80 \%$ acetonitrile) to $40 \% \mathrm{~B}$ in 120 minutes at $200 \mathrm{~nL} /$ minute then to $100 \% \mathrm{~B}$ in an additional 10 minutes. The high-performance liquid chromatography gradient was shallower than that of general proteomic analysis because phosphopeptides are relatively hydrophilic. Before and after analyzing one sample, the column was washed with high-performance liquid chromatography mobile phase B for 30 minutes, then mobile phase
A for 20 minutes at a high flow rate $(1 \mu \mathrm{L} /$ minute) to reduce potential carryover. The LTQ-Orbitrap mass spectrometer was operated in a data-dependent mode in which each full MS scan (60,000 resolving power) was followed by eight MS/MS scans where the eight most abundant molecular ions were dynamically selected and fragmented in by collisioninduced dissociation using a normalized collision energy of $35 \%$. The fragmented ions were detected by LTQ. The dynamic exclusion time was 30 seconds, and the dynamic exclusion size was 200. The FT master scan preview mode, charge state screening, monoisotopic precursor selection, and charge state rejection were enabled so that only the $1+$, $2+$, and $3+$ ions were selected and fragmented by collisioninduced dissociation.

Tandem mass spectra collected by Xcalibur version 2.0.2 were searched against the National Center for Biotechnology Information human protein database (released in September 2009 with 37,391 entries) using Proteome Discoverer software version 2.1 (Thermo Fisher Scientific) with full tryptic cleavage constraints, static cysteine alkylation by iodoacetamide, variable methionine oxidation, and variable phosphorylation of Ser/Thr/Tyr. Mass tolerance for precursor ions was $5 \mathrm{ppm}$, and mass tolerance for fragment ions was $0.25 \mathrm{Da}$. The Proteome Discoverer search results were filtered by criteria Xcorr versus charge 1.5, 1.8, 2.5 for $1+, 2+, 3+$ ions; ranked top \#1; probability of randomized identification of peptide $<0.1$. Confident peptide identification were determined using these stringent filter criteria for database match scoring followed by manual evaluation of the results. The false discovery rate was estimated by searching a combined forward-reversed database as described by Elias and Gygi. ${ }^{12}$ The MS proteomics data have been deposited to the ProteomeXchange Consortium via the PRIDE partner repository with the data set identifier PXD013473. ${ }^{13}$

\section{Reverse-Phase Protein Microarray Analysis}

Cellular lysates were printed in triplicate onto nitrocellulosecoated slides (Grace Bio-labs, Bend, OR) using an Aushon 2470 arrayer (Aushon BioSystems, Billerica, MA). Before proceeding with immunostaining, each array was treated with Reblot antibody stripping solution (Chemicon, Temecula, CA) for 15 minutes and blocked in I-block solution (Tropix, Bedford, MA) for 1 hour to reduce nonspecific binding. Each array was probed with one primary antibody on an automatic Autostainer (Dako Cytomation, Carpinteria, CA) using the Catalyzed Signal Amplification System kit (Dako Cytomation). Antibody specificity was tested for single-band specificity and ligand induction via Western blot analysis. Fluorescent detection was achieved using the streptavidin-conjugated IRDye680 (LI-COR Biosciences, Lincoln, NE) according to the manufacturer's instructions. The total amount of protein contained in each sample was measured by Sypro Ruby Protein Blot Stain (Molecular Probes, Eugene, OR) as previously described. ${ }^{14}$ Images 
were acquired using the PowerScanner (TECAN, Mönnedorf, Switzerland), and spot intensity values were quantified using MicroVigene software version 5.1.0.0 (VigeneTech, Carlisle, MA) as previously described. ${ }^{14}$ The RPPA raw data from J82 and T24 bladder cancer cells are available in Supplemental Tables S1 and S2, respectively.

\section{Immunofluorescence Microscopy}

Immunofluorescence staining of bladder cancer cells grown on poly-L-lysine coated glass coverslips was performed using standard procedures. Cells were fixed with ice-cold methanol for 10 minutes and blocked with $10 \%$ normal goat serum (Gibco) in PBS with $0.1 \%$ Triton X-100 for 30 minutes at room temperature. Cav-1 was stained using an Alexa Fluor 488-conjugated mouse anti-Cav-1 monoclonal antibody (1:10; catalog number IC5736G; R\&D Systems) for 1 hour at room temperature. Coverslips were mounted onto glass slides using ProLong Gold Antifade mountant with DAPI for nuclear counterstaining (Molecular Probes). Images were obtained on a Leica DM IRE2 inverted fluorescent microscope (Buffalo Grove, IL) with a Hamamatsu ORCA-100 digital camera (Sewickley, PA) using SimplePCI software version 6 (Hamamatsu Corp.).

\section{Electron Microscopy}

Preparation of cell lysates for transmission electron microscopy was performed using standard procedures. J82 cells were collected by trypsinization and fixed with Karnovsky's fixative $(2.5 \%$ glutaraldehyde and $4 \%$ paraformaldehyde in $0.1 \mathrm{~mol} / \mathrm{L}$ PBS, pH 7.4) at $4^{\circ} \mathrm{C}$ for a minimum of 3 hours. After rinsing with PBS, cells were postfixed in $1.5 \%$ osmium tetroxide in PBS for 45 minutes. Cells were then washed with deionized water and dehydrated in ascending concentrations (35\% to $100 \%$ ) of ethanol for 15 minutes each. Samples were then immersed in propylene oxide, 1:1 propylene oxide, and epoxy resin mixture (Quetol 651 embedding kit; Electron Microscopy Sciences, Hatfield, PA), 2:3 propylene oxide and epoxy resin mixture, and then overnight at $75^{\circ} \mathrm{C}$ with pure epoxy resin mixture under constant agitation and vacuum to optimize specimen infiltration. Thin sections $(60 \mathrm{~nm})$ were then cut using an RMC MTX-L ultra-microtome (Boeckeler Instruments, Inc., Tucson, AZ) and mounted on thin-bar 200-mesh copper grids. The grids were then stained with methanolic uranyl acetate saturated solution followed by bismuth subnitrate staining for contrast enhancement. Samples were examined with a Zeiss EM $10 \mathrm{C}$ transmission electron microscope (Oberkochen, Germany) and images captured using a GATAN model 785 digital camera (Pleasanton, CA).

\section{Human Samples}

Formalin-fixed, paraffin-embedded tumor blocks from 53 patients were curatively obtained following University of
Table 1 Clinicopathologic Features

\begin{tabular}{lc}
\hline Feature & All patients \\
\hline Age at diagnosis, years & \\
Mean, median & $65.9,64$ \\
Range & $43-84$ \\
Sex, No./Total No. (\%) & \\
$\quad$ Male & $39 / 53(74)$ \\
Female & $14 / 53(26)$ \\
Signs and/or symptoms, No./Total No. (\%)* & \\
Hematuria & $30 / 53(57)$ \\
$\quad$ Microhematuria & $2 / 53(3.8)$ \\
$\quad$ Gross hematuria & $28 / 53(53)$ \\
Obstruction & $3 / 53(5.7)$ \\
Irritative bladder & \\
Other (pain, weight loss) & $12 / 53(23)$ \\
None & $4 / 53(7.5)$ \\
Pathological T stage, No./Total No. (\%) & $15 / 53(28)$ \\
pT1 & \\
pT2 & $1 / 53(1.9)$ \\
pT3 & $13 / 53(25)$ \\
pT4 & $32 / 53(60)$ \\
Tumor size, cm & \\
Mean, median & $7 / 53(13)$ \\
Range & \\
\hline
\end{tabular}

*In many cases, patients reported multiple signs and/or symptoms.

†Iritative bladder signs and symptoms include frequency, urgency, dysuria, nocturia, polyuria, and urinary tract infection.

California, San Diego Institutional Review Board approval. Invasive disease (pT2+) was enriched in this patient population. Clinicopathologic information for this patient cohort is provided in Table 1.

\section{Immunohistochemistry}

Immunohistochemical staining of sectioned human tissue with Cav-1 (catalog number 3267; Cell Signaling) was performed using procedures as previously described. ${ }^{6}$ Briefly, tissue sections from paraffin-embedded blocks were cut onto precoated slides, followed by deparaffinization, rehydration, and heat-induced antigen retrieval using sodium citrate buffer. Sections were then blocked with $10 \%$ normal goat serum in TBS for 1 hour at room temperature and then incubated overnight at $4^{\circ} \mathrm{C}$ with primary antibody against Cav-1 diluted in blocking buffer. After four washes in TBS, blocking of endogenous peroxidase was performed by incubation with $3 \% \mathrm{H}_{2} \mathrm{O}_{2}$ in TBS for 10 minutes. For enzymatic detection, tissue was counterstained with speciesappropriate prediluted horseradish peroxidase polymer secondary antibody and chromagen developed with diaminobenzidine using a Rabbit/Mouse specific horseradish peroxidase/diaminobenzidine (ABC) Detection IHC Kit according to the manufacturer's instructions (AbCam).

\section{Ingenuity Pathway Analysis Analysis}

The Ingenuity Pathway Analysis program (Qiagen, Venlo, Netherlands) was used to analyze phosphoproteins that were 
Table 2 Phosphoproteins Identified by Mass Spectrometry Analysis Putatively Regulated by mTORC2

\begin{tabular}{ll}
\hline Protein & Gene \\
\hline Increased target protein phosphorylation by mTORC2 & \\
BCL2-associated transcription factor 1 isoform1 & BCLAF1 \\
Bifunctional coenzyme A synthase & COASY \\
Liprin $\alpha$-1* & PPFIA1 \\
Multiple PDZ domain protein & MPDZ \\
Myotubularin-related protein 14 & MTMR14 \\
Probable E3 ubiquitin-protein ligase HERC1 & HERC1 \\
R3H domain-containing protein 2 & R3HDM2 \\
Staphylococcal nuclease domain-containing & SND1 \\
protein 1 & \\
Talin-1* & TLN1 \\
Telomeric repeat-binding factor & TERF2IP \\
2-interacting protein 1 & \\
Decreased target protein phosphorylation by mTORC2 & \\
60S acidic ribosomal protein P0 & RPLPO \\
AMFR* & AMFR \\
Coiled-coil domain containing 6 & CCDC6 \\
cAMP-dependent transcription factor ATF-2 & ATF2 \\
Liprin $\beta$-1* & PPFIBP1 \\
Mediator of DNA damage checkpoint & MDC1 \\
protein 1 & \\
P44/42 mitogen-activated protein kinase 1 & MAPK1 \\
Protein scribble homolog & SCRIB \\
Solute carrier family 35, family C2 isoform b & SLC35C2 \\
Transcription factor AP-1 & JUN \\
Transcription factor ELYS & AHCTF1 \\
Transmembrane protein 43 & TMEM43 \\
\hline & \\
&
\end{tabular}

*Protein was validated by immunoblot analysis. All phosphoproteins were identified in at least two of three biological replicates.

AMFR, autocrine motility factor receptor; mTORC2, mammalian target of rapamycin complex 2 .

elevated in the control or RICTOR knockdown group. $P<$ 0.05 was used to select differentially expressed phosphoproteins. The shortlisted phosphoprotein names were then mapped to corresponding Entrez gene identification numbers. Both pathway and gene ontology enrichment analyses were conducted to identify candidates involved in some type of molecular and cellular component function as classified by the gene ontology nomenclature.

\section{Data Mining}

The cBio Portal for Cancer Genomics web tool was used to analyze the provisional RNA sequencing data from The Cancer Genome Atlas (TCGA; https://portal.gdc.cancer. gov/projects/TCGA-BLCA, last accessed January 25, 2019) for bladder urothelial carcinoma. This study includes 408 tumor samples. All searches were performed according to the cBioPortal instructions. ${ }^{15,16}$

\section{Statistical Analysis}

Tests for statistical significance were determined using the $t$-test with $P<0.05$. Comparison between Kaplan-Meier survival curves was performed using the Mantel-Cox log rank test. All statistical analyses were performed using GraphPad Prism software version 6 (GraphPad Software, La Jolla, CA).

\section{Results}

\section{MS-Based Analytics to Identify mTORC2 Targets}

Pharmacologic inhibitors of mTOR, such as PP242, KU0063795, and Torin-2, have served as valuable tools to study various biological processes associated with phosphatidylinositol 3-kinase (PI3K)/AKT/mTOR signaling. ${ }^{17,18}$ However, these inhibitors affect both mTOR-containing complexes, making it difficult to distinguish mTORC1versus mTORC2-mediated effects. Accordingly, targeted gene silencing of RICTOR was performed to selectively ablate mTORC2 activity, which was confirmed by reduced AKT S473 phosphorylation. This approach does not alter mTORC1-dependent phosphorylation of downstream signaling effectors. ${ }^{4}$ A migration model was implemented whereupon serum stimulation after serum starvation was used to induce cell migration, and this was compared with nonmotile, serum-starved cells transfected with siNTC. ${ }^{4} \mathrm{MS}$ was used as a phosphoproteomic discovery approach to capture global changes of phosphorylation events in these cell populations that were increased or decreased by the presence or absence of mTORC2. After downstream workflows, stringent filter criteria from MS analysis identified 212 and 199 unique peptides in control and rictorsilenced cells, respectively. Ten unique phosphoproteins in control siRNA-transfected cells and 12 unique phosphoproteins in rictor-silenced were identified by MS analysis in at least two of the three biological replicates (Table 2).

Ingenuity Pathway Analysis was used to perform functional enrichment of these proteins to determine the biological and/or signaling pathways affected by the presence or absence of mTORC2 activity. Ingenuity Pathway Analysis analysis revealed 26 and 22 significantly altered $(P<0.05)$ cellular functions up-regulated or downregulated by mTORC2 activity, respectively (Figure 1, A and B). Among these, the top five categories up-regulated by mTORC2 activity were cellular assembly and organization, cell death and survival, cellular growth and proliferation, cellular function and maintenance, and gene expression. By contrast, the top five most overrepresented categories down-regulated by mTORC2 activity included cell death and survival, cellular growth and proliferation, cellular development, cell morphology, and cellular assembly and organization.

Given the role of mTORC2 in regulating cytoskeletal remodeling, cell adhesion, and cell motility, the investigation was focused on the novel targets of mTORC2 for phosphoproteins involved in cellular assembly and organization, cell morphology, and cellular movement. Among these functions, mTORC2 increased phosphorylation of 
A Cellular Functions Increased by mTORC2 Activity

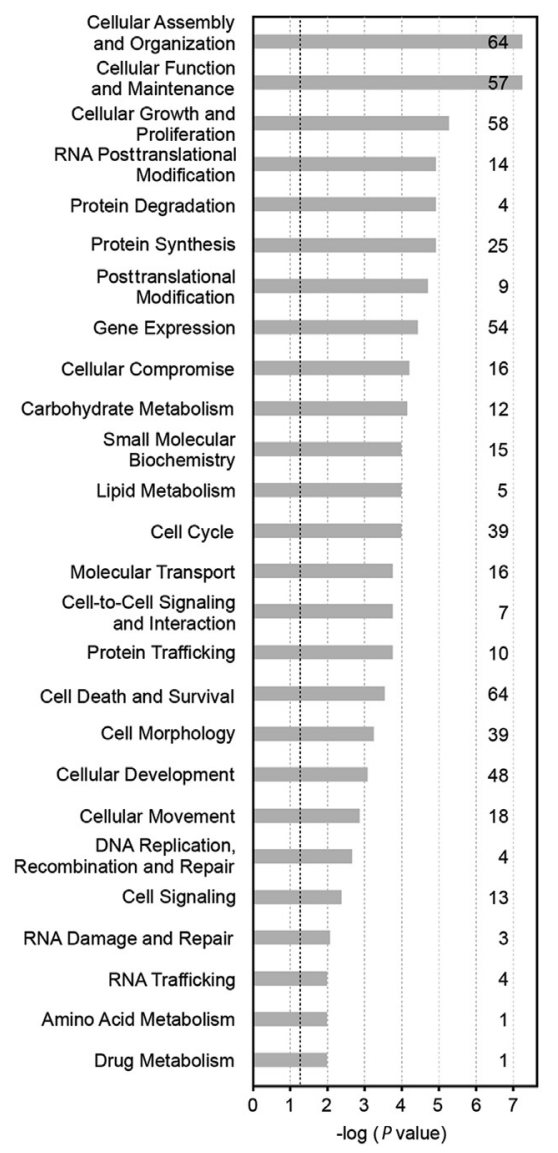

B Cellular Functions Decreased by mTORC2 Activity

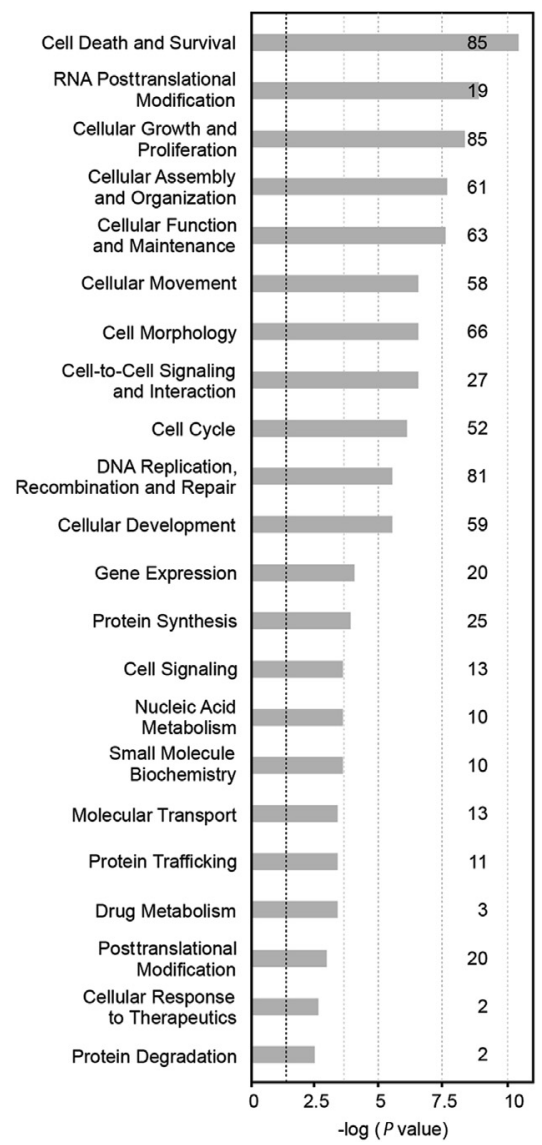

Figure 1 Mass spectrometry-based analytics to identify mammalian target of rapamycin complex 2 (mTORC2) targets. Ingenuity Pathway Analysis identified 26 and 22 significantly altered cellular functions putatively increased $(\mathbf{A})$ or decreased (B) by mTORC2 activity, respectively. The values adjacent to each bar indicate the number of phosphoproteins represented in each respective cellular function. See also Table 1. eight proteins and decreased phosphorylation of 32 proteins (Figure 2A and Table 3). One protein, nonmuscle myosin heavy chain 10, was identified in both categories. The Search Tool for the Retrieval of Interacting Genes/Proteins (STRING) database was used to assemble a protein interaction network of the 39 commonly represented phosphoproteins. ${ }^{19}$ Thirty-six phosphoproteins formed one large distinct network with significantly enriched interactions (Figure 2B). Two of the top five gene ontology biological processes that were significantly enriched within this network included movement of cell or subcellular components $\left(n=19 / 35 ; P=1.26 \times 10^{-9}\right)$ and regulation of cell motility $\left(n=14 / 35 ; P=7.76 \times 10^{-9}\right)$.

\section{Protein Pathway Activation Mapping}

Toward the goal of identifying novel signaling effectors of mTORC2 activity, the differential protein and phosphoprotein changes that occur within the bladder cancer cell migration model were examined using RPPA analysis. This method serves as a complementary approach to MS and the global analysis of mTORC2 signaling perturbations in the rictor-silenced cell migration model. Lysates were probed with $\geq 150$ antibodies that represented a diverse set of signaling pathways, biological functions, and protein classes, including PI3K/AKT/mTOR signaling, mitogenactivated protein kinase (MAPK)/extracellular-signal-regulated kinase (ERK) signaling, Janus kinase (JAK)/STAT signaling, AMP-activated protein kinase (AMPK) signaling and energy sensing, PKC signaling, cell stress and immune response, focal adhesion and cell motility, cell cycle regulation, cell death and apoptosis, translation and transcription, tumor suppressors and oncogenes, and receptor tyrosine kinases (RTKs) (Supplemental Figure S1). Rictor silencing and consequent loss of mTORC2 activity decreased AKT S473 phosphorylation, whereas downstream mTORC1 targets (p-AKT T308, p-PRAS40 T246, p-S6 S235/236 and S240/244, p-p70-S6K S371, T389 and T412, and p-4EBP1 S65 and T70) were unaffected in serumstimulated cells (Figure 3).

RPPA analysis also identified that mTORC2 can regulate additional phosphoprotein targets across multiple signaling pathways. Loss of mTORC2 activity resulted in activation of cell death and apoptosis-signaling effectors, including caspases 3, 6, 7, and 9, poly (ADP-ribose) polymerase, and increased phosphorylation of several members of the Bcl-2 family of apoptosis proteins, which confirmed previous reports linking mTORC2 with apoptosis in leukemic, breast, and non-small cell lung carcinoma cell line models. $^{20-22}$ By contrast, reduced pathway activation of 

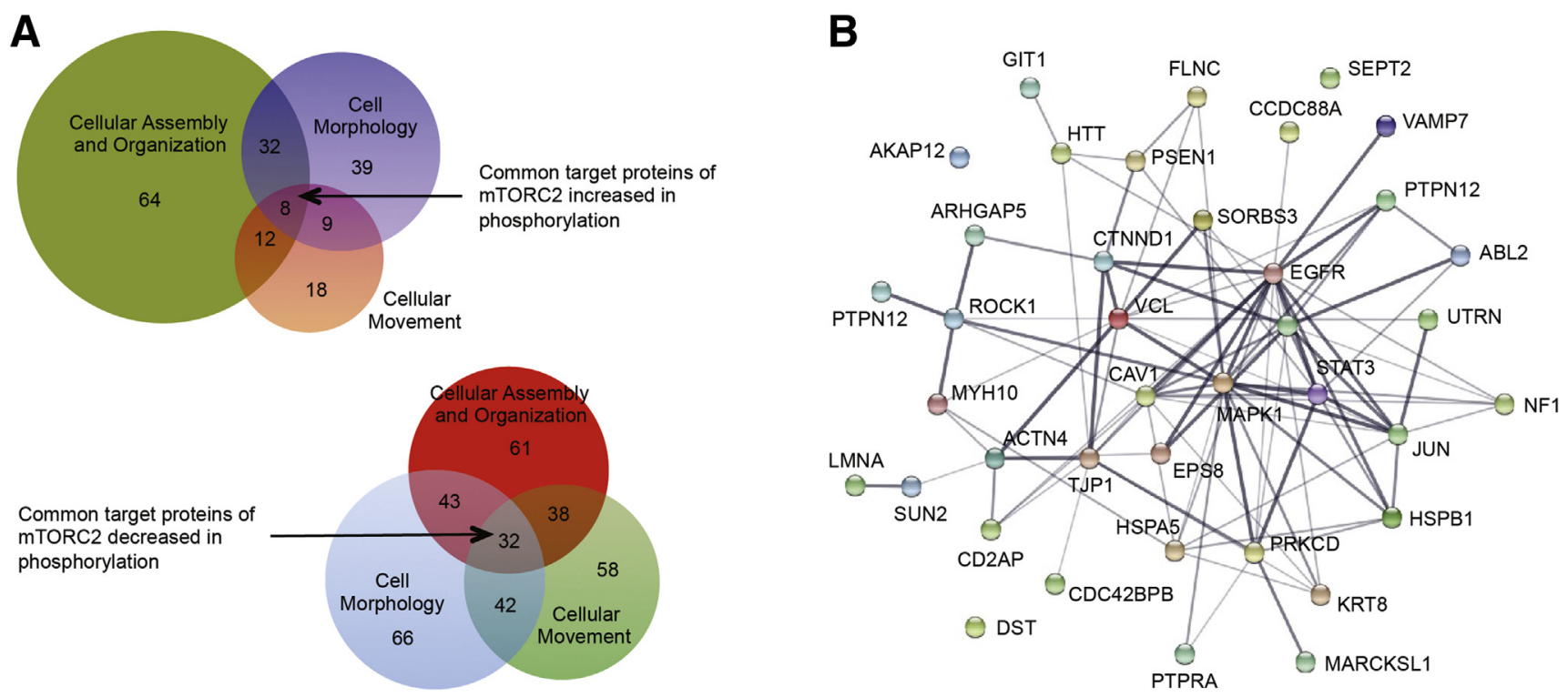

Figure 2 Pathway and gene ontology analysis of mammalian target of rapamycin complex 2 (mTORC2)-regulated targets. A: Venn diagrams depicting the number of phosphoproteins represented among the indicated functions in control or rictor-silenced cells, respectively. B: Common unique phosphoproteins queried through the STRING database reveals one large protein interaction network. The Search Tool for the Retrieval of Interacting Genes/Proteins (STRING) database assembles information about known and predicted protein interactions from numerous sources. Thicker lines represent stronger associations between proteins. ABL1, tyrosine-protein kinase ABL1; ABL2, Abelson tyrosine protein kinase 2; ACTN4, alpha-actinin-4; AKAP12, A-kinase anchor protein 12; ARHGAP5, Rho GTPase-activating protein 5; ARHGEF12, Rho guanine nucleotide exchange factor 12; CAV1, caveolin-1; CCDC88A, girdin; CD2AP, CD2-associated protein; CDC42BPB, serine/threonine-protein kinase MRCK beta; CTNND1, catenin (cadherin-associated protein), D1; DST, dystonin; EGFR, epidermal growth factor receptor; EPS8, Abl interactor 1; FLNC, filamin C, gamma; GIT1, ARF GTPase-activating protein GIT1; HSPA5, 78-kDa glucose-regulated protein; HSPB1, heat shock protein beta-1; HTT, huntingtin; JUN, transcription factor AP-1; KRT8, keratin, type II cytoskeletal 8; LMNA, prelamin-A/C; MAPK1, mitogen-activated protein kinase 1; MARCKSL1, MARCKS-related protein; MYH10, myosin, heavy chain 10, nonmuscle; NF1, neurofibromin; PRKCD, protein kinase C, delta; PSEN1, presenilin1; PTPN12, tyrosine-protein phosphatase nonreceptor type 12; PTPRA, receptor-type tyrosine-protein phosphatase alpha; ROCK1, Rho-associated protein kinase 1; SEPT2, septin-2; SORBS3, vinexin; STAT3, signal transducer and activator of transcription 3; SUN2, SUN domain-containing protein 2; UTRN, utrophin; VAMP7, vesicle-associated membrane protein 7; TJP1, tight junction protein Z0-1; VCL, vinculin.

numerous signaling effectors associated with the MAPK/ ERK, JAK/STAT, AMPK, and PKC pathways, as well as proteins involved in cell motility and focal adhesion (p-adducin S662, p-cofilin S3, p-CrkII Y221, and p-SRC Y416), cell cycle regulation (cyclins A and D1, p-Chk-1 $\mathrm{S} 345$, and $\mathrm{p}-\mathrm{Rb} \mathrm{S} 780$ ), and transcription and translation (p-eIF2a S51, p-eIF4E S209, and p-FKHR S256 and T24) was observed.

\section{Validation of mTORC2 Effects on Discovery Phosphoprotein Targets}

To validate the findings from parallel MS and RPPA analysis, a large subset of proteins and phosphoproteins was selected to confirm the directional change in expression or phosphorylation status between siNTC or siRictortransfected bladder cancer cells through immunoblot analysis under nonmotile (serum starved) and motile (serum stimulated) conditions in J82 and T24 bladder cancer cells. $^{23,24}$ The effects of mTORC2 signaling were confirmed on known targets to verify the specificity of rictor silencing in selectively reducing mTORC2 activity. Rictor silencing ablated mTORC 2 activity as evidenced by reduced phosphorylation of AKT S473 (Figure 3A). In addition,
mTORC1 phosphorylation targets were unaffected by rictor silencing, with no phosphorylation alterations evident in AKT T308, S6 235/236 and S240/244, p70-S6K T389, and 4EBP1 T37/46, consistent with data from the RPPA analysis and prior studies. ${ }^{4}$

Given the previously described role of mTORC2 in regulating bladder cancer cell migration and invasion, immunoblot validation was performed to confirm the effects of mTORC2driven expression and phosphorylation changes of proteins known or proposed to be associated with these cellular processes. $^{4-6}$ In some cases, discrepancies between RPPA and representative immunoblot signals may be the result of using different antibodies with different epitope specificities and/or interassay variation. Loss of rictor affected the expression and/ or phosphorylation of proteins involved in cell motility and focal adhesion, many of which have not been previously associated with mTORC2 activity (Figure 3B). In rictor-ablated cells, increased expression or phosphorylation of the cell adhesion factors FAK Y397, Y576/577 and Y925, total FAK, $\mathrm{N}$-cadherin, $\beta$-catenin, $\mathrm{p}$-ezrin T567, total talin-1, and snail was observed in both serum-starved and serum-stimulated conditions when compared with NTC control cells. By contrast, expression of liprin $\beta-1$ and TJP1/ZO- 1 and phosphorylation of cofilin S3 were increased in rictor-silenced cells in the absence 

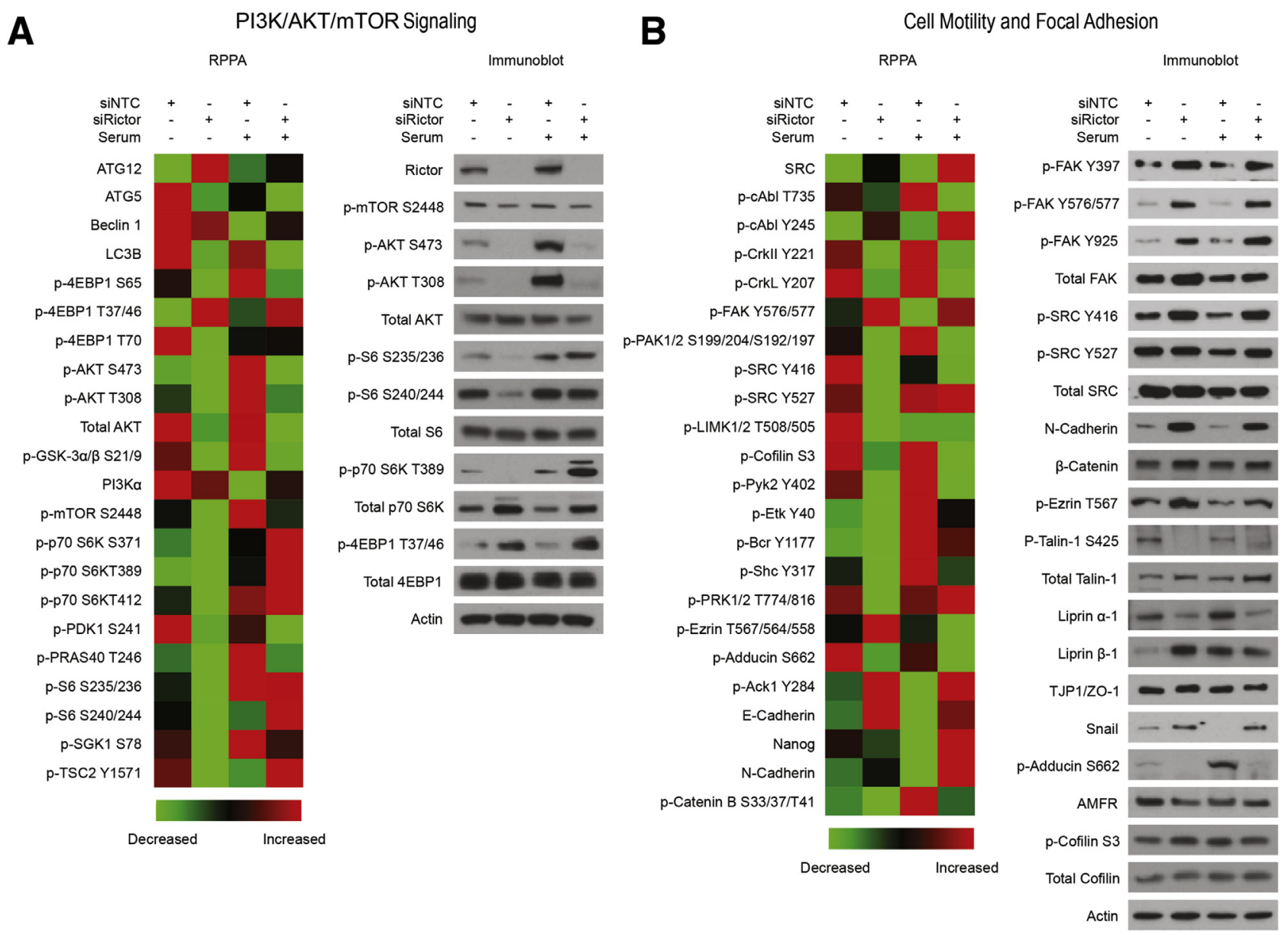

Figure 3 Protein pathway activation mapping of mammalian target of rapamycin complex 2 (mTORC2) ablated J82 bladder cancer cells. Validation of mass spectrometry and reverse-phase protein array (RPPA) results by immunoblot analysis for proteins and phosphoproteins involved in phosphatidylinositol 3kinase, AKT, and mTOR signaling (A) and cell motility and focal adhesion (B). Selected proteins and phosphoproteins representing their respective signaling pathway or cellular processes from RPPA analysis are also shown by each heat map for side-by-side comparison. All immunoblots are representative of at least three independent experiments. See also Supplemental Figure S1. 4EBP1, eukaryotic translation initiation factor 4E binding protein 1; Ack1, activated CDC42 Kinase 1; AMFR, autocrine motility factor receptor; ATG12, autophagy related 12; ATG5, autophagy related 5; Bcr, BCR activator of RhoGEF and GTPase; cAbl, c-Abl oncogene 1, receptor tyrosine kinase; CrkII, CRK proto-oncogene, adaptor protein; CrkL, CRK-like proto-oncogene, adaptor protein; Etk, epithelial and endothelial tyrosine kinase; FAK, focal adhesion kinase; GSK-3 alpha/beta, glycogen synthase kinase-3 alpha/beta; LC3B, microtubule associated protein 1 light chain 3 beta; LIMK1/2, LIM domain kinase 1/2; mTOR, mammalian target of rapamycin; p-, phosphorylated; p70 S6K, ribosomal protein S6 kinase beta-1; PAK1/2, p21 (RAC1) activated kinase 2; PDK1, phosphoinositide-dependent kinase-1; PI3K alpha, phosphoinositide 3-kinase alpha; PRAS40, proline-rich AKT1 substrate 1; PRK1/2, protein kinase C-related kinase 1/2; Pyk2, protein-tyrosine kinase 2-beta; S6, ribosomal protein S6; SGK1, serum/glucocorticoid regulated kinase 1; Shc, Shc adaptor protein 1; siNTC, nontargeting control siRNA SRC, SRC proto-oncogene, non-receptor tyrosine kinase; TJP/Z0-1, tight junction protein/zona occludens 1; TSC2, tuberous sclerosis 2 protein.

of serum, suggesting differential effects of mTORC2 activity under motile versus nonmotile conditions. Loss of mTORC2 activity resulted in reduction of talin-1 S425 and adducin S662 phosphorylation and protein expression of liprin $\alpha-1$ and autocrine motility factor receptor in both serum-starved and stimulated conditions. These immunoblot findings are consistent with results obtained by RPPA analysis and represent validation of novel signaling effectors and downstream mediators of mTORC2. A subset of these proteins $(\mathrm{N}$-cadherin, $\beta$-catenin, snail, and TJP1/ZO-1) have been implicated in epithelialmesenchymal transition and with increased metastatic potential in a number of tumor systems, suggesting programmatic changes in cell function may also be regulated by
mTORC $2 .^{25-32}$ These findings were also validated in human T24 bladder cancer cells (Supplemental Figures S2 and S3).

\section{Phosphorylation of Cav-1 and Modification of Caveolae-Associated Receptor Tyrosine Kinases Are Regulated by mTORC2}

A major motivation of this study was to identify novel targets of mTORC 2 activity in the context of active bladder cancer cell migration. The unbiased global view of phosphoprotein expression changes due to mTORC2 silencing afforded by MS complemented by the high analytical sensitivity of RPPA to measure phosphorylation statuses of 
Table 3 Common Phosphoproteins Represented Among Cellular Assembly and Organization, Cell Morphology, and Cellular Movement as Determined by IPA Analysis

\begin{tabular}{|c|c|}
\hline Protein & Gene \\
\hline \multicolumn{2}{|l|}{ Represented in control cells } \\
\hline Caveolin-1* & CAV1 \\
\hline Serine/threonine-protein kinase MRCK $\beta$ & $C D C 42 B P B$ \\
\hline Catenin (cadherin-associated protein), $\Delta 1$ & CTNND1 \\
\hline Filamin $C, \gamma$ & FLNC \\
\hline Huntingtin & $H T T$ \\
\hline Myosin, heavy chain 10 , nonmuscle & MYH10 \\
\hline Protein kinase $\mathrm{C}, \Delta$ & $P R K C D$ \\
\hline Vesicle-associated membrane protein 7 & VAMP7 \\
\hline \multicolumn{2}{|l|}{ Represented in rictor-silenced cells } \\
\hline Tyrosine-protein kinase ABL1 & $A B L 1$ \\
\hline Abelson tyrosine-protein kinase 2 & $A B L 2$ \\
\hline$\alpha$-actinin-4 & ACTN4 \\
\hline A-kinase anchor protein 12 & AKAP12 \\
\hline Rho GTPase-activating protein 5 & ARHGAP5 \\
\hline Rho guanine nucleotide exchange factor 12 & ARHGEF12 \\
\hline Girdin & CCDC88A \\
\hline CD2-associated protein & $C D 2 A P$ \\
\hline Dystonin & DST \\
\hline Epidermal growth factor receptor* & EGFR \\
\hline Abl interactor 1 & EPS8 \\
\hline ARF GTPase-activating protein GIT1 & GIT1 \\
\hline 78-kDa glucose-regulated protein & HSPA5 \\
\hline Heat shock protein $\beta-1$ & HSPB1 \\
\hline Transcription factor AP-1 & JUN \\
\hline Keratin, type II cytoskeletal 8 & KRT8 \\
\hline Prelamin-A/C & LMNA \\
\hline Mitogen-activated protein kinase 1 & MAPK1 \\
\hline MARCKS-related protein & MARCKSL1 \\
\hline Myosin, heavy chain 10 , nonmuscle & MYH10 \\
\hline Neurofibromin & NF1 \\
\hline Presenilin-1 & PSEN1 \\
\hline $\begin{array}{l}\text { Tyrosine-protein phosphatase nonreceptor } \\
\text { type } 12\end{array}$ & PTPN12 \\
\hline $\begin{array}{l}\text { Receptor-type tyrosine-protein } \\
\text { phosphatase } \alpha^{*}\end{array}$ & PTPRA \\
\hline Rho-associated protein kinase 1 & ROCK1 \\
\hline Septin-2 & SEPT2 \\
\hline Vinexin & SORBS3 \\
\hline $\begin{array}{l}\text { Signal transducer and activator of } \\
\text { transcription } 3\end{array}$ & STAT3 \\
\hline SUN domain-containing protein 2 & SUN2 \\
\hline Tight junction protein $\mathrm{ZO}-\mathrm{1}^{*}$ & $T J P 1$ \\
\hline Utrophin & UTRN \\
\hline Vinculin & $V C L$ \\
\hline
\end{tabular}

*Denotes that protein was validated by immunoblot analysis. IPA, Ingenuity Pathway Analysis.

proteins relevant to cell motility and focal adhesion would allow for discovery of proteins using our bladder cancer cell migration model. To this end, MS analysis identified Cav-1 (Table 3) as a candidate downstream target of mTORC2 and is functionally associated with pathways relevant to cell migration, including cellular assembly and organization, cell morphology, and cellular movement. Cav-1 belongs to a family of integral membrane proteins and is an essential component of small (50 to $100 \mathrm{~nm}$ ) flask-shaped invaginations of the plasma membrane called caveolae. ${ }^{33}$ Aside from roles in caveolae formation and stability, Cav1 acts as a scaffolding protein within caveolae and noncaveolar subdomains of lipid rafts to cluster and regulate numerous signaling molecules, such as $\mathrm{G}$ proteins, integrins, SRC family tyrosine kinases, PI3Ks, and RTKs. ${ }^{33}$ On the basis of identification of an abundance of phosphorylated Cav-1 in rictor-expressing control cells using MS, upregulation of Cav-1 activity by mTORC 2 was anticipated. In particular, two well-characterized phosphorylation sites, Y14 and S80, were considered as possible residues that are potentially phosphorylated after serum stimulation of siNTC-transfected control cells. Residue Y14 phosphorylation is essential for Cav-1 association with SH2domain-containing adaptor proteins, such as Grb7, at focal adhesion sites that promote integrin-mediated cell migration. ${ }^{34,35}$ Phosphorylation of this residue is also required for caveolae-mediated endocytosis of cell surface signaling proteins.

To test whether Cav-1 Y14 is differentially phosphorylated in rictor-expressing versus rictor-silenced cells, immunoblot analysis of Y14 phosphorylation was performed in response to serum stimulation. A rapid and robust up-regulation of Cav-1 Y14 phosphorylation was observed after the addition of serum in rictor-expressing J82 cells as evidenced by immunoblot and corresponding densitometry analyses (Figure 4, A and B). By contrast, mTORC2silenced cells showed constitutive phosphorylation of Y14 in both the presence and absence of serum, suggesting that rictor knockdown results in loss of dynamic regulation of Cav-1. Given the effect of mTORC2 silencing on Cav-1 phosphorylation, the expression of cavin-1, a necessary component for caveolae formation, ${ }^{36}$ was subsequently investigated and its expression found to be markedly upregulated on rictor silencing and serum stimulation.

To verify the potential interaction of Cav-1 with mTORC2, mTORC2-associated proteins were immunoprecipitated from T24 and J82 bladder cancer cell lysates using a rictor antibody-Sepharose bead conjugate. Intact mTORC2 was confirmed based on co-immunoprecipitation of enriched amounts of mTOR and the mTORC2-specific component, mSIN1 (Figure 4C). In both cell lines, coimmunoprecipitation of Cav-1 was observed with rictor antibody, confirming an interaction between the mTORC2 complex and Cav-1.

Growth factor RTKs coordinate numerous biological processes and are subjected to multiple levels of regulation, including attenuation of receptor autophosphorylation by protein tyrosine phosphatases, receptor sequestration and degradation, and up-regulation of inhibitory proteins that counteract downstream signaling effectors. ${ }^{37}$ Both mTORC1 and mTORC2 signaling complexes coordinate negative feedback signals to some growth factor RTKs, 


\section{A}

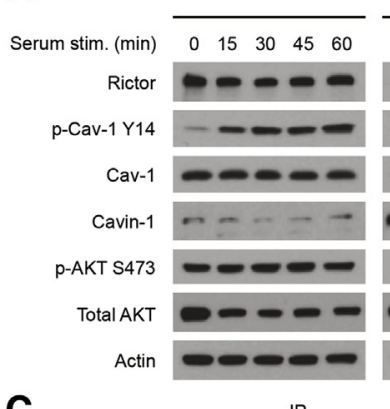

C

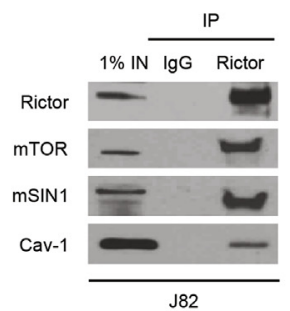

D

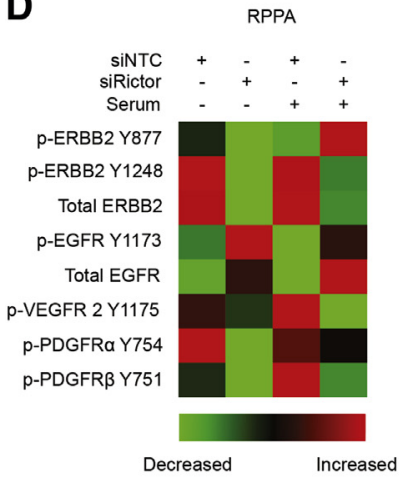

siRictor
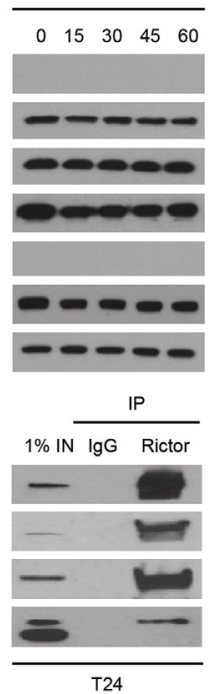

B
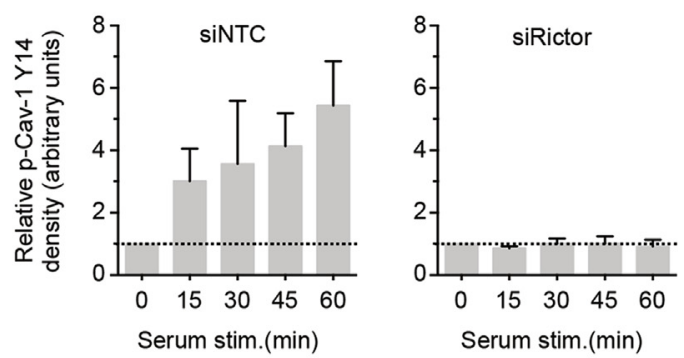

$\mathbf{E}$

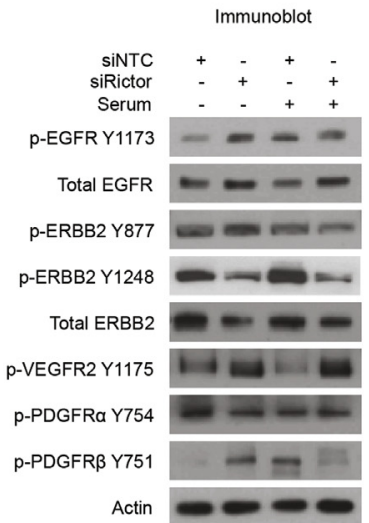

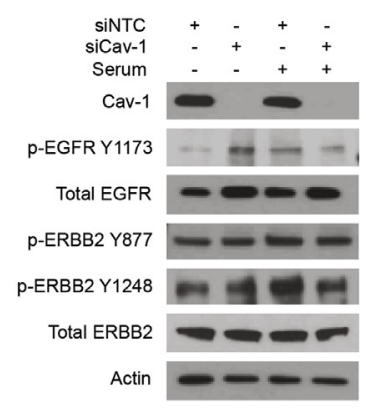

$\mathbf{F}$

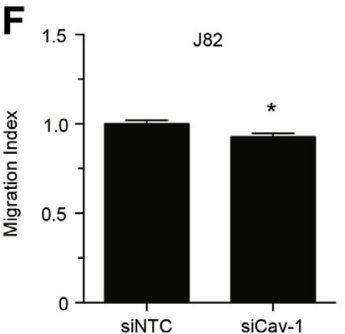

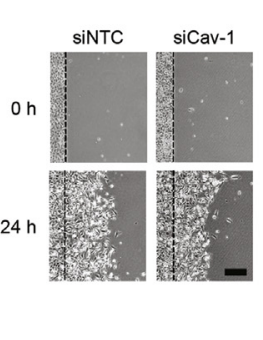

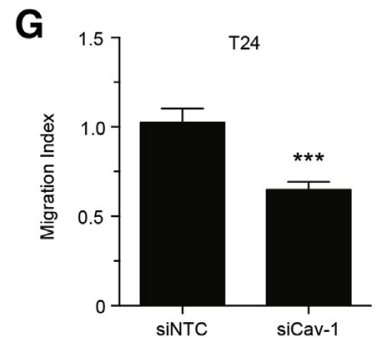

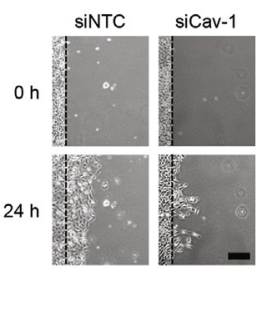

Figure 4 Phosphorylation of caveolin-1 (Cav-1) and modification of caveolae-associated receptor tyrosine kinases are regulated by mTORC2. A: 0ne-hour time-course serum stimulation of nontargeting control siRNA (siNTC) - or siRNA against RICTOR (siRictor)-transfected J82 cells to assess Cav-1 Y14 phosphorylation and cavin-1 protein expression levels. Phosphorylated (p)-AKT S473 levels were evaluated to confirm down-regulation of mammalian target of rapamycin complex 2 (mTORC2) activity. B: Corresponding densitometry analysis of p-Cav-1 Y14 expression. Dashed lines indicate normalized p-Cav1 Y14 signal density at 0 minutes. C: $\mathrm{J} 82$ and T24 cells were subjected to immunoprecipitation (IP) with an IgG isotype control or anti-rictor Sepharose bead conjugate, and immunoprecipitates were immunoblotted with the indicated antibodies. D and E: Co-IP of Cav-1 was observed with endogenous intact mTORC2. Immunoblot analyses of select growth factor receptors in response to rictor (D) or Cav-1 (E) silencing in J82 cells. All immunoblots are representative of at least three independent experiments. F and G: Representative micrographs and quantification of distance migrated of control (siNTC) and Cav-1-silenced J82 (F) and T24 (G) cells serum starved for 12 to 16 hours before serum stimulation to promote cell migration during 24 hours. Dashed lines indicate the migratory cell front at $t=0$ hours. Data are expressed as means \pm SE from three independent experiments (B) and means \pm SEM of 50 random measurements per field from three independent experiments (F and $\mathbf{G}) .{ }^{*} P<0.05,{ }^{* *} P<0.001$ (two-tailed $t$-test). Scale bar $=200 \mu \mathrm{m}$. EGFR, epidermal growth factor receptor; IN, input; mSIN1, stress-activated map kinase interacting protein 1; PDGFR, platelet-derived growth factor receptor; RPPA, reverse-phase protein array; VEGFR, vascular endothelial growth factor receptor.

such as insulin and insulin-like growth factor 1 receptors, PDGFR- $\beta$, and HER kinase receptors. ${ }^{38-42}$ Consistent with these reports, reduced phosphorylation status of Y1135/36 on insulin-like growth factor 1 receptor, Y751 and Y716 on PDGFR- $\beta$, and Y1248 on HER2 was detected in rictor-silenced J82 cells by RPPA and immunoblot analysis (Figure 4D and Supplemental Figure S1). Because Cav-1 functions as a scaffolding protein to 
A

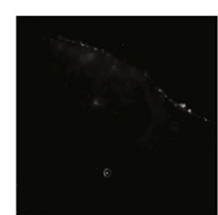

Cav-1

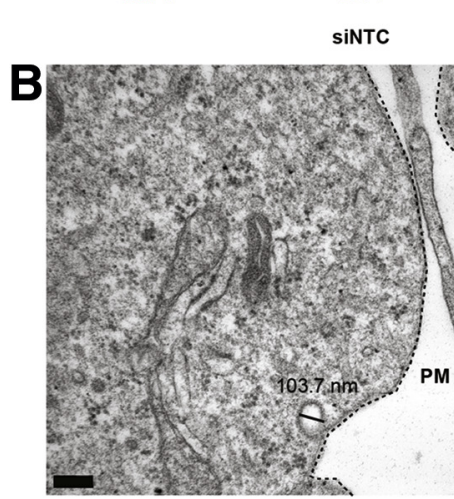

D

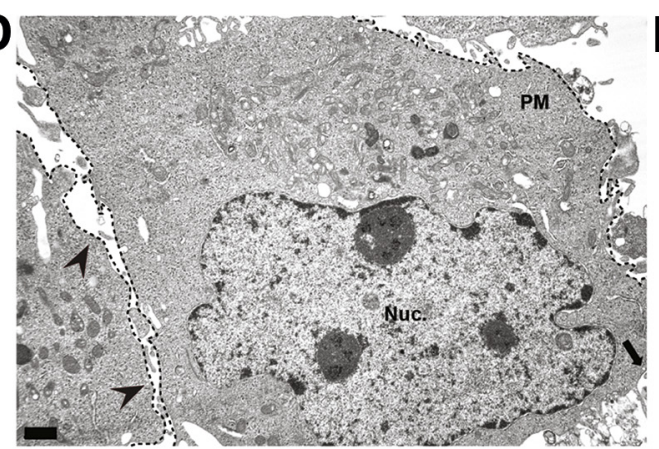

$F^{\circ}$

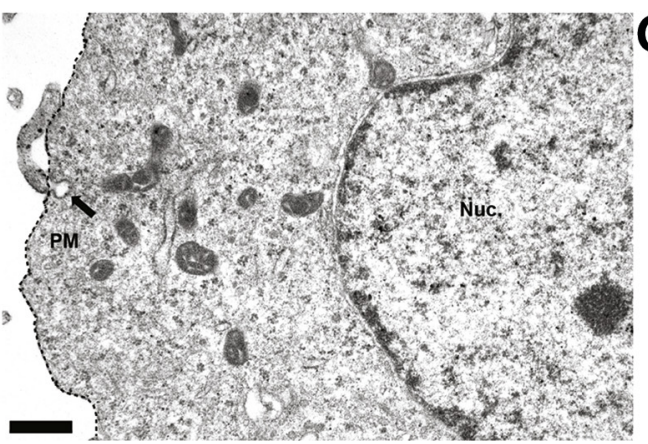

siNTC

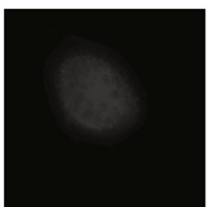

DAPI

Overlay

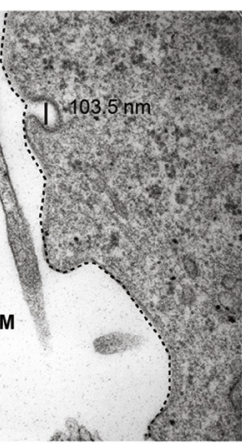

E

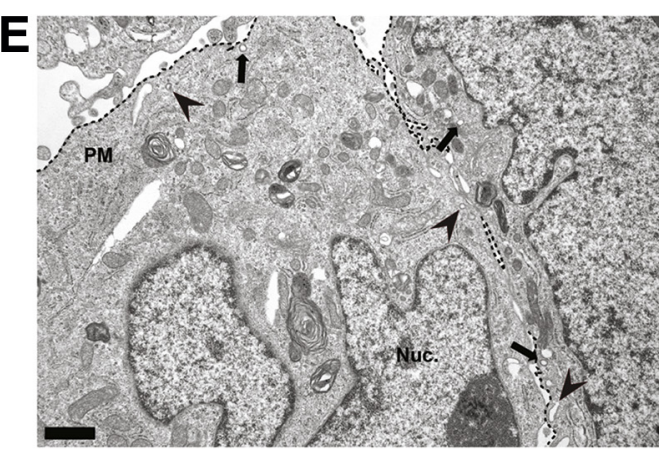

G

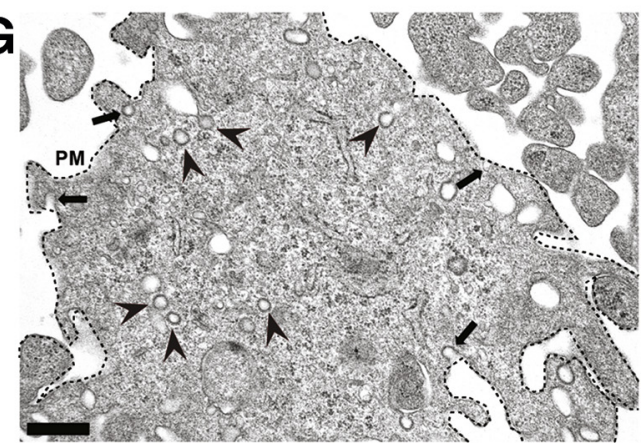

Figure 5 Mammalian target of rapamycin complex 2 (mTORC2) can induce caveolin-1 (Cav-1) redistribution and modify caveolae abundance. A: Nontargeting control siRNA (siNTC) - or siRNA against RICTOR (siRictor)-transfected J82 bladder cancer cells were grown on glass coverslips and stimulated with serum for 1 hour. Cells were then fixed for immunofluorescence staining of Cav-1. The overlay panels depict the merged images for Cav-1 (green) and DAPI (blue). B-G: Electron micrographs of siNTC (B, D and F) and rictor silenced (C, E and $\mathbf{G}$ ) cells. Caveolae are detected as flask-shaped invaginations of the plasma membrane (PM) and fully invaginated vesicles in the cytosol. Dotted lines outline the PM. Arrows denote invaginating caveolae on the PM and arrowheads indicate vesicular caveolae. Scale bars: $200 \mathrm{~nm}$ (B and C), $1 \mu \mathrm{m}$ (D and E), and $0.5 \mu \mathrm{m}$ (F and G). Nuc, nucleus.

regulate signal transduction and rictor silencing eliminates dynamic Cav-1 phosphorylation, we hypothesized that mTORC2 feedback may regulate RTKs through modulation of Cav-1 activity. The effect of Cav-1 silencing was therefore evaluated on the phosphorylation status of EGFR and HER2. No apparent differences in EGFR Y1173, HER2 Y877, and HER2 Y1248 phosphorylation and EGFR and HER2 expression were observed in Cav$1-$ silenced J82 cells compared with rictor silencing, suggesting that loss of mTORC2 activity may directly affect regulation of EGFR and $\mathrm{HER} 2$ through $\mathrm{Cav}-1$ (Figure 4E).

Lastly, the role of Cav-1 was evaluated on bladder cancer cell migration using a 24-hour modified scratch-wound migration assay. Cav-1 silencing had a small but significant (8\%; $P=0.01$, two-tailed $t$-test) inhibitory effect on $\mathrm{J} 82$ cell migration (Figure $4 \mathrm{~F}$ ) in contrast to a modest and significant decrease $(35 \% ; P=0.0002$, two-tailed $t$-test) in 
T24 cells (Figure 4G). These results are consistent with previous reports establishing a promigratory role of Cav-1 in bladder cancer cells. ${ }^{43-45}$

\section{mTORC2 Can Induce Cav-1 Redistribution and Modify Caveolae Abundance}

Whereas tyrosine-14 phosphorylation of Cav-1 is linked to the regulation of cell signaling and caveolae-mediated endocytosis, serine- 80 phosphorylation localizes Cav-1 to endoplasmic reticulum membranes. ${ }^{46}$ Because commercial antibodies are not currently available for immunoblot detection of p-Cav-1 S80 that would allow directly determining whether this residue is activated by mTORC2, immunofluorescence microscopy was used to determine the localization pattern of Cav-1 in response to mTORC2 ablation and serum stimulation. In both control and rictor-silenced cells, a punctate staining pattern of Alexa Fluor 488-labeled Cav-1 was present throughout the cell (Figure 5A). However, in only control cells, Cav-1 was also localized to the edge and rear of cells in a manner consistent with polarization of Cav-1 during cell migration. ${ }^{47}$ Together, these results suggest that the Y14 and not the S80 residue of Cav-1 may be modified by mTORC2 signaling because a reticular staining pattern consistent with endoplasmic reticulum localization was not observed in either experimental condition.

Because the data showed increased cavin-1 expression, modification of Y14 Cav-1 phosphorylation, and redistribution of Cav-1 with rictor silencing, we hypothesized that loss of mTORC 2 activity might also alter the abundance of caveolae. Transmission electron microscopy was used to evaluate changes in caveolae abundance in mTORC2-ablated J82 cells. In both NTC and rictor siRNA-transfected cells, membrane-dense flask-shaped invaginations were observed on the plasma membrane and vesicular and fully-invaginated caveolae within the cell cytosol (Figure 5, B and C). The size of these vesicles (approximately $100 \mathrm{~nm}$ ) was consistent with caveolae, as previously described. ${ }^{48}$ However, compared with rictor-expressing control cells (Figures 5, D and F), an increased abundance of caveolae was observed in rictorsilenced cells (Figures 5, E and G), suggesting that mTORC2 activity affects caveolae formation through regulation of cavin-1 expression and/or Cav-1 Y14 phosphorylation.

\section{Association of Caveolin-1 with Overall Survival in Human Bladder Cancer}

Having identified Cav-1 as a potential target of mTORC2, which can promote bladder cancer cell invasion, ${ }^{4} \mathrm{Cav}-1$ expression was analyzed in a cohort of patients with urothelial carcinoma to determine its association with outcomes. Previous studies have found that increased Cav-1 expression correlates with increasing bladder tumor grade and stage, ${ }^{49,50}$ although there are limited reports of Cav-1
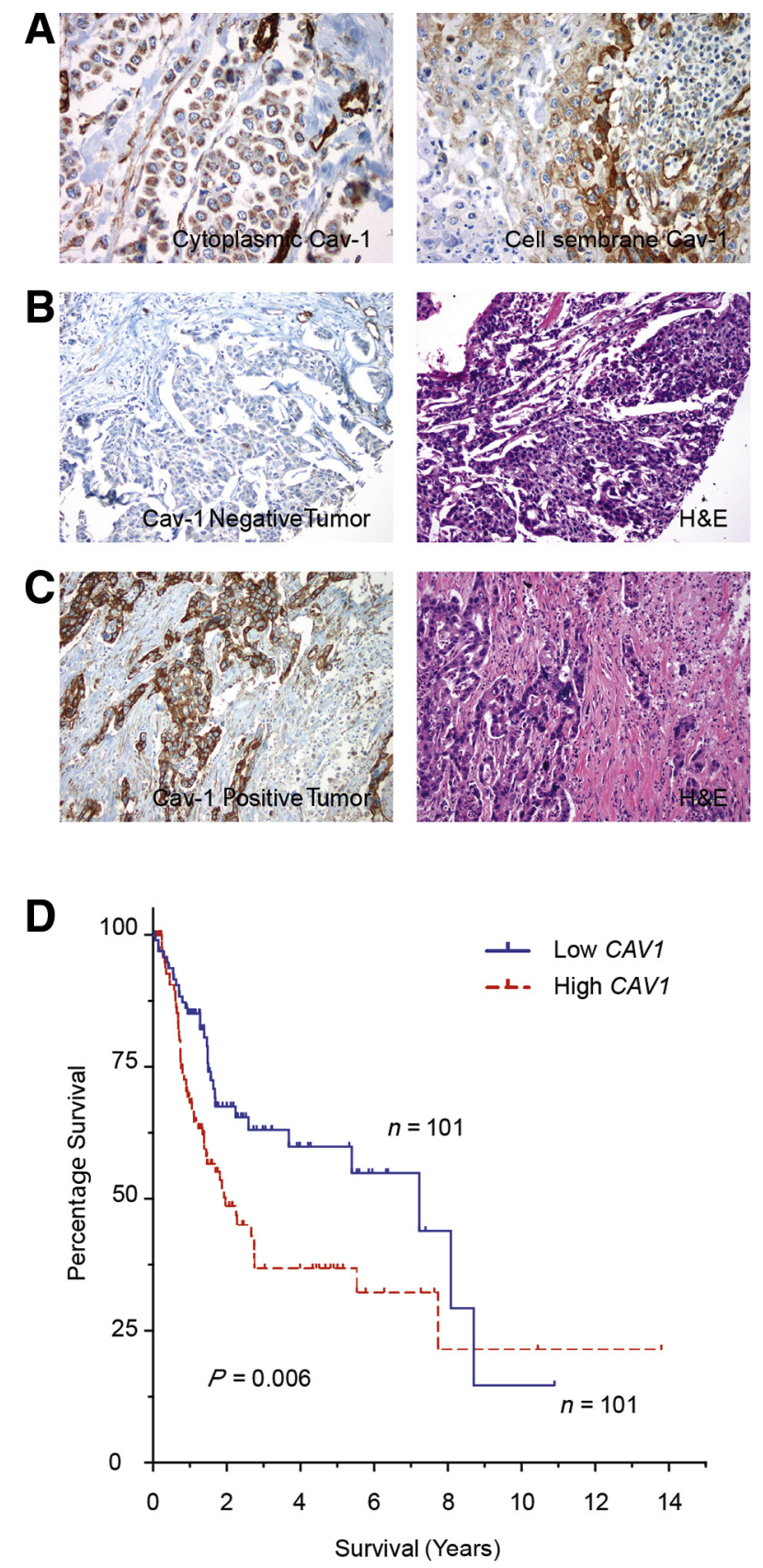

Figure 6 Caveolin-1 (Cav-1) is expressed in high-grade invasive bladder cancer. Immunohistochemical staining was performed with an anti-Cav-1 monoclonal antibody. A: Cav-1 positivity is primarily observed as diffuse cytoplasmic staining though membranous staining is present in some cases. B and C: Negative Cav-1 tumor immunoreactivity with matching hematoxylin and eosin (H\&E) staining (B) in contrast to positive tumor expression (C). D: Kaplan-Meier analysis showing association between CAV1 mRNA expression and overall survival based on the provisional data set from The Cancer Genome Atlas on bladder cancer. The top and bottom quartiles of CAV1 expression among all patients were defined as high and low CAV1 expressors, respectively. A significant difference in overall survival is observed between high and low CAV1 expression. $P=0.006$.

association with outcomes. Cav-1 expression was assessed using immunohistochemistry in 53 primary high-grade bladder cancers that were associated with metastasis in a subset of cases. Cav-1 was expressed in the cell cytoplasm 
and/or cell membrane in 49 of 53 cases (92.5\%) (Figure 6A). The remaining four patients $(7.5 \%)$ lacked detectable Cav-1 expression. Representative images with matching hematoxylin and eosin stains of Cav-1-negative and -positive specimens are shown in Figure 6, B and C.

It was next tested whether Cav-1 expression can predict patient outcomes. High Cav-1 expression was associated with a 5-year survival rate of $18.5 \%$ compared with a $46.1 \%$ 5-year survival rate associated with low Cav-1 expression. The publicly available transcriptomics bladder cancer data from the provisional TCGA RNA sequencing data set were next examined. High and low CAVI expression $(n=101$ each) was defined as the top and bottom quartiles, respectively, among the patient cohort $(n=408)$. A significant reduction in overall survival $(P=0.006)$ was observed in patients with high $C A V I$ expression compared with low $C A V 1$ expression, with median survival rates of 2.0 and 7.2 years, respectively (Figure 6D).

\section{Discussion}

mTOR plays a central role in cellular growth and proliferation, metabolism, and cell motility through two distinct multiprotein complexes, mTORC1 and mTORC2. Given its significance in maintaining cellular homeostasis, it is fitting that dysregulation of mTOR activity is associated with myriad malignancies, including bladder cancer. Although much is known about the regulation and functions of mTORC1, studies that define mTORC2 activity are more limited. Our laboratory has previously described a critical role for mTORC2 in promoting bladder cancer migration and invasion, with downstream effects on RhoA and Rac1 likely responsible in part for mediating this response. ${ }^{4}$ To identify additional potential targets of mTORC2 signaling during bladder cancer motility, MS- and RPPA-based proteomic analysis was used concomitantly. Diverse categories of phosphoproteins pivotal in a wide range of cellular processes were identified, including those involved in cell motility, such as autocrine motility factor receptor; cytoskeletal arrangement, such as adducin and cofilin; and cell and focal adhesion, including FAK, SRC, N-cadherin, $\beta$-catenin, ezrin, talin-1, liprin $\alpha-1$, liprin $\beta-1$, TJP1/ZO-1, and snail. Cav-1 was also identified as a novel downstream target of $\mathrm{mTORC} 2$, with mTORC2 regulation of Cav-1 phosphorylation and localization contributing to altered expression of known cancerassociated RTKs, such as EGFR and focal adhesion complexes, which were also identified through our screen.

Cav-1 has diverse functions that can positively or negatively regulate cell signaling pathways. For example, both EGFR and the insulin receptor can be negatively and positively activated by Cav-1 expression. ${ }^{51-53}$ The differential regulatory roles of Cav-1 on cell surface receptors underscore the complexity of Cav-1 involvement in disease and biological processes as additionally evidenced by the variable effects on bladder cancer cell migration observed when Cav-1 is silenced. Cav-1 was first described as a direct substrate of SRC kinase that localizes to caveolae. ${ }^{54}$ Subsequent studies also revealed the localization of Cav-1 to noncaveolar lipid rafts at or near focal adhesion sites where Y14-phosphorylated Cav-1 regulates focal adhesion complex dynamics to control directional migration. ${ }^{34}$ The identification of an enrichment of phosphorylated Cav-1 in the rictor-expressing control bladder cancer cell model by MS suggested that mTORC2 could regulate Cav-1 activity through phosphorylation of the Y14 and/or S80 residues. Co-immunoprecipitation of Cav-1 was observed with mTORC2, and immunoblot analysis showed that Cav-1 phosphorylation on Y14 was robustly up-regulated after serum-stimulation in rictor-expressing control cells and could be ablated when rictor was silenced. However, this does not exclude the possibility of an indirect interaction between mTORC 2 and Cav-1.

To further support the hypothesis that mTORC2 regulates Cav-1, the subcellular distribution pattern of Cav-1 was analyzed in cells with or without rictor silencing after serum stimulation to promote cell motility. In serum-stimulated control cells, Cav-1 was frequently polarized to the membrane and trailing edge of cells. By contrast, in rictorsilenced cells, Cav-1 staining appeared punctate within the cell cytosol with no apparent evidence of endoplasmic reticulum localization indicative of S80 phosphorylation. ${ }^{46,47}$ Therefore, the observed difference in Cav-1 polarization is likely the result of Y14 rather than S80 phosphorylation. However, this assessment of mTORC2 regulation of S80 phosphorylation is limited because of the lack of a commercial antibody to this site. Given the observed localization of Cav-1 to the plasma membrane under motile conditions in control cells and the mTORC2-driven cavin- 1 expression change identified with rictor silencing, whether mTORC2 could regulate formation or abundance of caveolae in this model system was tested. Rictor silencing resulted in increased caveolae formation seen by transmission electron microscopy. On the basis of these data, mTORC2 appears to be an upstream mediator of Cav-1 Y14 phosphorylation and may be responsible for regulating caveolae formation and abundance. Because caveolae can cluster and enhance the signaling of numerous RTKs within the cell, mTORC2 may also have a role in mediating bladder cancer caveolarassociated RTK protein expression and/or stability, including EGFR and HER2.

Cell migration is a complex process that requires spatial and temporal regulation of numerous signaling components at integrin-mediated adhesion sites. These adhesions dynamically form and turn over at the leading edge of a cell to generate traction forces while they disassemble at the rear to produce rear-end retraction and detachment for cell body translocation. This highly regulated process is understood to be primarily mediated by FAK and SRC tyrosine kinases that signal to numerous downstream effectors, including Cav-1 and the Rho family GTPases Rac1 and RhoA, the latter of which was previously found to be regulated by 
mTORC2 in bladder cancer cells. ${ }^{4,55}$ Under serum-free conditions in which cells do not migrate, Y14 of Cav-1 was not phosphorylated in control cells but was markedly phosphorylated on serum stimulation. Because mTORC2related expression changes in focal adhesion complex proteins were seen, whether mTORC 2 could spatially regulate Cav-1 localization to cell adhesions to prevent focal adhesion turnover, which thereby maintains a resting (nonmotile) state, was examined. This theory is supported by findings reported by Beardsley et $\mathrm{al}^{47}$ that suggest that loss of Cav-1 polarity in stationary cells and targeted silencing of Cav-1 impede directional movement. The authors also found that immunofluorescence staining for Cav-1 in stationary cells appeared punctate throughout the cell, a similar result observed in rictor-silenced, serum-stimulated bladder cancer cells.

Prior studies have failed to demonstrate an association between Cav-1 expression and overall survival for patients with bladder cancer, ${ }^{49,50}$ although the patient numbers were limited. Public gene expression data from the provisional TCGA bladder cancer study that includes a large patient data set were therefore examined. A highly significant association was found between high $C A V 1$ expression and reduced median and overall survival.

In summary, two parallel phosphoproteomics approaches were used to identify protein and phosphoprotein targets of mTORC2, including regulators of cell motility, cell morphology, cellular assembly and organization, and several other functions that have not been previously linked with mTORC2 activity. mTORC2 was found to dynamically regulate Cav-1 Y14 phosphorylation, alter Cav-1 localization, and mediate caveolae formation. Although known to regulate some components of focal adhesion complexes, additional cell adhesion and cell motility proteins were also identified as potential targets of mTORC2, and these proteins may be regulated in conjunction with Cav-1. Specifically, the effects on the activities of FAK and $\mathrm{SRC}$ on rictor silencing would suggest a functional association among these kinases and mTORC2. Indeed, a link between mTORC2 activation and Fyn, a SRC family kinase, in cooperation with FAK was previously described during focal adhesion signaling that defines marrow-derived mesenchymal stem cell fate and cytoskeletal structure emanating from mechanical stimuli, such as cell stress or strain. ${ }^{56,57}$ However, it is unclear whether the same association exists during growth factor-induced activation of mTORC2. Future studies that dissect the role of mTORC2 in the regulation of the caveolar microenvironment are important to determine effects on RTK signaling in the context of cell motility and stasis and the regulation of additional proteins associated with focal adhesions.

\section{Acknowledgments}

We thank Dr. Henry C. Powell (University of California, San Diego) for his assistance and expert advice for the electron microscopy experiments, Drs. David M. Sabatini (Massachusetts Institute of Technology) and Dudley W. Lamming (University of Wisconsin) for technical protocols for rictor co-immunoprecipitation, and Dr. Kun-Liang Guan (University of California, San Diego) for critically reviewing the manuscript.

A.M.H., S.G., S.M., E.F.P., and D.E.H. designed research; A.M.H., S.G., M.Z.L., K.N., J.M., W.Z., A.H., and J.W. performed research; B.C., K.B., and S.R. analyzed data; A.M.H, K.B., S.R, S.M., E.F.P., and D.E.H. reviewed and/or wrote the article; and D.E.H. is the guarantor of this work and, as such, had full access to all of the data in the study and takes responsibility for the integrity of the data and the accuracy of the data analysis.

\section{Supplemental Data}

Supplemental material for this article can be found at https://doi.org/10.1016/j.ajpath.2019.05.010.

\section{References}

1. American Cancer Society: Cancer Facts \& Figures 2018. Atlanta, American Cancer Society, 2018

2. Babjuk M, Bohle A, Burger M, Capoun O, Cohen D, Comperat EM, Hernandez V, Kaasinen E, Palou J, Roupret M, van Rhijn BW, Shariat SF, Soukup V, Sylvester RJ, Zigeuner R: EAU guidelines on non-muscle-invasive urothelial carcinoma of the bladder: update 2016. Eur Urol 2017, 71:447-461

3. Kamat AM, Colombel M, Sundi D, Lamm D, Boehle A, Brausi M Buckley R, Persad R, Palou J, Soloway M, Witjes JA: BCG-unresponsive non-muscle-invasive bladder cancer: recommendations from the IBCG. Nat Rev Urol 2017, 14:244-255

4. Gupta S, Hau AM, Beach JR, Harwalker J, Mantuano E, Gonias SL, Egelhoff TT, Hansel DE: Mammalian target of rapamycin complex 2 (mTORC2) is a critical determinant of bladder cancer invasion. PLoS One 2013, 8:e81081

5. Gupta S, Hau AM, Al-Ahmadie HA, Harwalkar J, Shoskes AC Elson P, Beach JR, Hussey GS, Schiemann WP, Egelhoff TT, Howe PH, Hansel DE: Transforming growth factor-beta is an upstream regulator of mammalian target of rapamycin complex 2dependent bladder cancer cell migration and invasion. Am J Pathol 2016, 186:1351-1360

6. Hau AM, Leivo MZ, Gilder AS, Hu JJ, Gonias SL, Hansel DE mTORC2 activation is regulated by the urokinase receptor (UPAR) in bladder cancer. Cell Signal 2017, 29:96-106

7. Saxton RA, Sabatini DM: mTOR signaling in growth, metabolism, and disease. Cell 2017, 169:361-371

8. Oh WJ, Jacinto E: mTOR complex 2 signaling and functions. Cell Cycle 2011, 10:2305-2316

9. Pierobon M, Wulfkuhle J, Liotta L, Petricoin E: Application of molecular technologies for phosphoproteomic analysis of clinical samples. Oncogene 2015, 34:805-814

10. Sarbassov DD, Ali SM, Kim DH, Guertin DA, Latek RR, ErdjumentBromage H, Tempst P, Sabatini DM: Rictor, a novel binding partner of mTOR, defines a rapamycin-insensitive and raptor-independent pathway that regulates the cytoskeleton. Curr Biol 2004, 14: 1296-1302

11. Zhou W, Capello M, Fredolini C, Piemonti L, Liotta LA, Novelli F, Petricoin EF: Mass spectrometry analysis of the post-translational modifications of alpha-enolase from pancreatic ductal adenocarcinoma cells. J Proteome Res 2010, 9:2929-2936 
12. Elias JE, Gygi SP: Target-decoy search strategy for increased confidence in large-scale protein identifications by mass spectrometry. Nat Methods 2007, 4:207-214

13. Perez-Riverol Y, Csordas A, Bai J, Bernal-Llinares M, Hewapathirana S, Kundu DJ, Inuganti A, Griss J, Mayer G, Eisenacher M, Perez E, Uszkoreit J, Pfeuffer J, Sachsenberg T, Yilmaz S, Tiwary S, Cox J, Audain E, Walzer M, Jarnuczak AF, Ternent T, Brazma A, Vizcaino JA: The PRIDE database and related tools and resources in 2019: improving support for quantification data. Nucleic Acids Res 2019, 47:D442-D450

14. Wulfkuhle JD, Berg D, Wolff C, Langer R, Tran K, Illi J, Espina V, Pierobon M, Deng J, DeMichele A, Walch A, Bronger H, Becker I, Waldhor C, Hofler H, Esserman L, Investigators IST, Liotta LA, Becker KF, Petricoin EF 3rd: Molecular analysis of HER2 signaling in human breast cancer by functional protein pathway activation mapping. Clin Cancer Res 2012, 18:6426-6435

15. Gao J, Aksoy BA, Dogrusoz U, Dresdner G, Gross B, Sumer SO, Sun Y, Jacobsen A, Sinha R, Larsson E, Cerami E, Sander C, Schultz N: Integrative analysis of complex cancer genomics and clinical profiles using the cBioPortal. Sci Signal 2013, 6:p11

16. Cerami E, Gao J, Dogrusoz U, Gross BE, Sumer SO, Aksoy BA, Jacobsen A, Byrne CJ, Heuer ML, Larsson E, Antipin Y, Reva B, Goldberg AP, Sander C, Schultz N: The cBio cancer genomics portal: an open platform for exploring multidimensional cancer genomics data. Cancer Discov 2012, 2:401-404

17. Xie J, Wang X, Proud CG: mTOR inhibitors in cancer therapy. F1000Res 2016, 5

18. Hau AM, Nakasaki M, Nakashima K, Krish G, Hansel DE: Differential mTOR pathway profiles in bladder cancer cell line subtypes to predict sensitivity to mTOR inhibition. Urol Oncol 2017, 35: 593-599

19. Szklarczyk D, Franceschini A, Wyder S, Forslund K, Heller D, Huerta-Cepas J, Simonovic M, Roth A, Santos A, Tsafou KP, Kuhn M, Bork P, Jensen LJ, von Mering C: STRING v10: proteinprotein interaction networks, integrated over the tree of life. Nucleic Acids Res 2015, 43:D447-D452

20. Li H, Lin J, Wang X, Yao G, Wang L, Zheng H, Yang C, Jia C, Liu A, Bai X: Targeting of mTORC2 prevents cell migration and promotes apoptosis in breast cancer. Breast Cancer Res Treat 2012, 134:1057-1066

21. Zhao L, Yue P, Khuri FR, Sun SY: mTOR complex 2 is involved in regulation of Cbl-dependent c-FLIP degradation and sensitivity of TRAIL-induced apoptosis. Cancer Res 2013, 73:1946-1957

22. Carayol N, Vakana E, Sassano A, Kaur S, Goussetis DJ, Glaser H, Druker BJ, Donato NJ, Altman JK, Barr S, Platanias LC: Critical roles for mTORC2- and rapamycin-insensitive mTORC1-complexes in growth and survival of BCR-ABL-expressing leukemic cells. Proc Natl Acad Sci U S A 2010, 107:12469-12474

23. Bubenik J, Baresova M, Viklicky V, Jakoubkova J, Sainerova H, Donner J: Established cell line of urinary bladder carcinoma (T24) containing tumour-specific antigen. Int J Cancer 1973, 11:765-773

24. O’Toole C, Price ZH, Ohnuki Y, Unsgaard B: Ultrastructure, karyology and immunology of a cell line originated from a human transitional-cell carcinoma. Br J Cancer 1978, 38:64-76

25. Lamouille S, Xu J, Derynck R: Molecular mechanisms of epithelialmesenchymal transition. Nat Rev Mol Cell Biol 2014, 15:178-196

26. Clucas J, Valderrama F: ERM proteins in cancer progression. J Cell Sci 2014, 127:267-275

27. Desiniotis A, Kyprianou N: Significance of talin in cancer progression and metastasis. Int Rev Cell Mol Biol 2011, 289:117-147

28. Wiener JR, Kerns BJ, Harvey EL, Conaway MR, Iglehart JD, Berchuck A, Bast RC Jr: Overexpression of the protein tyrosine phosphatase PTP1B in human breast cancer: association with p185cerbB-2 protein expression. J Natl Cancer Inst 1994, 86:372-378

29. Bravo-Cordero JJ, Magalhaes MA, Eddy RJ, Hodgson L, Condeelis J: Functions of cofilin in cell locomotion and invasion. Nat Rev Mol Cell Biol 2013, 14:405-415
30. Astro V, Asperti C, Cangi MG, Doglioni C, de Curtis I: Liprin-alpha1 regulates breast cancer cell invasion by affecting cell motility, invadopodia and extracellular matrix degradation. Oncogene 2011, 30: $1841-1849$

31. Matsuoka Y, Li X, Bennett V: Adducin: structure, function and regulation. Cell Mol Life Sci 2000, 57:884-895

32. Funasaka T, Raz A: The role of autocrine motility factor in tumor and tumor microenvironment. Cancer Metastasis Rev 2007, 26:725-735

33. Williams TM, Lisanti MP: The caveolin proteins. Genome Biol 2004, 5:214

34. Lee H, Volonte D, Galbiati F, Iyengar P, Lublin DM, Bregman DB, Wilson MT, Campos-Gonzalez R, Bouzahzah B, Pestell RG, Scherer PE, Lisanti MP: Constitutive and growth factor-regulated phosphorylation of caveolin-1 occurs at the same site (Tyr-14) in vivo: identification of a c-Src/Cav-1/Grb7 signaling cassette. Mol Endocrinol 2000, 14:1750-1775

35. Volonte D, Galbiati F, Pestell RG, Lisanti MP: Cellular stress induces the tyrosine phosphorylation of caveolin-1 (Tyr(14)) via activation of p38 mitogen-activated protein kinase and c-Src kinase. Evidence for caveolae, the actin cytoskeleton, and focal adhesions as mechanical sensors of osmotic stress. J Biol Chem 2001, 276:8094-8103

36. Hill MM, Bastiani M, Luetterforst R, Kirkham M, Kirkham A, Nixon SJ, Walser P, Abankwa D, Oorschot VM, Martin S, Hancock JF, Parton RG: PTRF-Cavin, a conserved cytoplasmic protein required for caveola formation and function. Cell 2008, 132:113-124

37. Lemmon MA, Schlessinger J: Cell signaling by receptor tyrosine kinases. Cell 2010, 141:1117-1134

38. Woo SY, Kim DH, Jun CB, Kim YM, Haar EV, Lee SI, Hegg JW, Bandhakavi S, Griffin TJ, Kim DH: PRR5, a novel component of mTOR complex 2, regulates platelet-derived growth factor receptor beta expression and signaling. J Biol Chem 2007, 282: $25604-25612$

39. Zhang H, Bajraszewski N, Wu E, Wang H, Moseman AP, Dabora SL, Griffin JD, Kwiatkowski DJ: PDGFRs are critical for PI3K/Akt activation and negatively regulated by mTOR. J Clin Invest 2007, 117:730-738

40. Ozes ON, Akca H, Mayo LD, Gustin JA, Maehama T, Dixon JE, Donner DB: A phosphatidylinositol 3-kinase/Akt/mTOR pathway mediates and PTEN antagonizes tumor necrosis factor inhibition of insulin signaling through insulin receptor substrate-1. Proc Natl Acad Sci U S A 2001, 98:4640-4645

41. O'Reilly KE, Rojo F, She QB, Solit D, Mills GB, Smith D, Lane H, Hofmann F, Hicklin DJ, Ludwig DL, Baselga J, Rosen N: mTOR inhibition induces upstream receptor tyrosine kinase signaling and activates Akt. Cancer Res 2006, 66:1500-1508

42. Chandarlapaty S, Sawai A, Scaltriti M, Rodrik-Outmezguine V, Grbovic-Huezo O, Serra V, Majumder PK, Baselga J, Rosen N: AKT inhibition relieves feedback suppression of receptor tyrosine kinase expression and activity. Cancer Cell 2011, 19:58-71

43. Thomas S, Overdevest JB, Nitz MD, Williams PD, Owens CR, Sanchez-Carbayo M, Frierson HF, Schwartz MA, Theodorescu D: Src and caveolin-1 reciprocally regulate metastasis via a common downstream signaling pathway in bladder cancer. Cancer Res 2011 , $71: 832-841$

44. Zhou W, He L, Dai Y, Zhang Y, Wang J, Liu B: MicroRNA-124 inhibits cell proliferation, invasion and migration by targeting CAV1 in bladder cancer. Exp Ther Med 2018, 16:2811-2820

45. Liang W, Hao Z, Han JL, Zhu DJ, Jin ZF, Xie WL: CAV-1 contributes to bladder cancer progression by inducing epithelial-tomesenchymal transition. Urol Oncol 2014, 32:855-863

46. Schlegel A, Arvan P, Lisanti MP: Caveolin-1 binding to endoplasmic reticulum membranes and entry into the regulated secretory pathway are regulated by serine phosphorylation. Protein sorting at the level of the endoplasmic reticulum. J Biol Chem 2001, 276:4398-4408

47. Beardsley A, Fang K, Mertz H, Castranova V, Friend S, Liu J: Loss of caveolin-1 polarity impedes endothelial cell polarization and directional movement. J Biol Chem 2005, 280:3541-3547 
48. Palade GE: Fine structure of blood capillaries. J Appl Phys 1953, 24:1424

49. Rajjayabun PH, Garg S, Durkan GC, Charlton R, Robinson MC, Mellon JK: Caveolin-1 expression is associated with high-grade bladder cancer. Urology 2001, 58:811-814

50. Fong A, Garcia E, Gwynn L, Lisanti MP, Fazzari MJ, Li M: Expression of caveolin-1 and caveolin-2 in urothelial carcinoma of the urinary bladder correlates with tumor grade and squamous differentiation. Am J Clin Pathol 2003, 120:93-100

51. Yamamoto M, Toya Y, Schwencke C, Lisanti MP, Myers MG Jr, Ishikawa Y: Caveolin is an activator of insulin receptor signaling. J Biol Chem 1998, 273:26962-26968

52. Couet J, Sargiacomo M, Lisanti MP: Interaction of a receptor tyrosine kinase, EGF-R, with caveolins. Caveolin binding negatively regulates tyrosine and serine/threonine kinase activities. J Biol Chem 1997, 272:30429-30438
53. Repetto S, Salani B, Maggi D, Cordera R: Insulin and IGF-I phosphorylate eNOS in HUVECs by a caveolin-1 dependent mechanism. Biochem Biophys Res Commun 2005, 337:849-852

54. Glenney JR Jr: Tyrosine phosphorylation of a $22-\mathrm{kDa}$ protein is correlated with transformation by Rous sarcoma virus. J Biol Chem 1989, 264:20163-20166

55. Mitra SK, Hanson DA, Schlaepfer DD: Focal adhesion kinase: in command and control of cell motility. Nat Rev Mol Cell Biol 2005, 6:56-68

56. Thompson WR, Guilluy C, Xie Z, Sen B, Brobst KE, Yen SS, Uzer G, Styner M, Case N, Burridge K, Rubin J: Mechanically activated Fyn utilizes mTORC2 to regulate RhoA and adipogenesis in mesenchymal stem cells. Stem Cells 2013, 31:2528-2537

57. Sen B, Xie Z, Case N, Thompson WR, Uzer G, Styner M, Rubin J: mTORC2 regulates mechanically induced cytoskeletal reorganization and lineage selection in marrow-derived mesenchymal stem cells. J Bone Miner Res 2014, 29:78-89 